Preprint typeset in JHEP style. - HYPER VERSION

hep-th/0204004

LPTENS-02-22

LPTHE-02-18

\title{
Strings in homogeneous gravitational waves and null holography
}

\author{
E. Kiritsis \\ Laboratoire de Physique Théorique de l'Ecole Normale Supérieure \\ 24 rue Lhomond, Paris, CEDEX 05, F-75231, FRANCE \\ and \\ Department of Physics, University of Crete, and FO.R.T.H. \\ 71003 Heraklion, GREECE \\ E-mail: kiritsis@physics.uoc.gr

\section{B. Pioline \\ LPTHE, Universités Paris VI et VII, 4 pl Jussieu, 75252 Paris cedex 05, FRANCE} \\ E-mail: pioline@lpthe.jussieu.fr
}

\begin{abstract}
Homogeneous gravitational wave backgrounds arise as infinite momentum limits of many geometries with a well-understood holographic description. General global aspects of these geometries are discussed. Using exact CFT techniques, strings in pp-wave backgrounds supported by a Neveu-Schwarz flux are quantized. As in Euclidean $A d S_{3}$, spectral flow and associated long strings are shown to be crucial in obtaining a complete spectrum. Holography is investigated using conformally flat coordinates analogous to those of the Poincaré patch in AdS. It is argued that the holographic direction is the light-cone coordinate $u$, and that the holographic degrees of freedom live on a codimension-one screen at fixed $u$. The usual conformal symmetry on the boundary is replaced by a representation of a Heisenberg-type algebra $H_{D} \times H_{D}$, hinting at a new class of field theories realizing this symmetry. A sample holographic computation of 2 and 3-point functions is provided and Ward identities are derived. A complementary screen at fixed $v$ is argued to be necessary in order to encode the vacuum structure.
\end{abstract}

KEYwORDS: Holography, pp-wave, light-cone quantization, Heisenberg algebra. 


\section{Contents}

1. Introduction 2

2. Geometry of homogeneous wave backgrounds 5

2.1 The homogeneous wave as a group manifold 5

2.2 Isometries and geodesics 7

2.3 Geodesics and waves

2.4 Representation theory of the extended Heisenberg group $H_{D}$

3. Strings in Neveu-Schwarz homogeneous pp-waves 11

3.1 Chiral primaries and vertex operators 12

3.2 Spectral flow and long strings 13

3.3 String spectrum

3.4 The fate of discrete symmetries 17

4. Null holography 17

4.1 Holography in the Poincaré patch 18

4.2 Geometry of conformal coordinates 19

4.3 Generalized conformal wave background 20

4.4 Null Cauchy problem 21

4.5 Boundary symmetries and Ward identities 23

4.6 Two-point and three-point functions 26

5. Summary, conclusions and open problems 27

A. Propagators 31

A.1 Boundary and bulk to bulk propagators in global coordinates 31

A.2 Propagators in conformal coordinates 32

B. The general scalar solution to the wave equation 34

C. Holography in the Poincaré patch 37

D. Solution of the Ward Identities 38

E. Holography in the harmonic oscillator basis 41 


\section{Introduction}

Despite the success of the AdS/CFT correspondence and the recent progress in understanding black hole entropy and holographic bounds, the concept of holography still lacks a general background independent formulation (see e.g. [1] for recent reviews). In the standard anti-de Sitter case, progress is hindered by the difficulty in quantizing strings in Ramond backgrounds. For de Sitter the existence of stable string backgrounds is itself a problem, due to the lack of supersymmetry. Flat Minkowski space has neither of these problems, yet its null conformal boundary makes it hard to formulate holography. Recently, this problem has taken a new turn with the realization that a particular scaling limit [2] of the $A d S_{5} \times S^{5}$ background, namely zooming on a null trajectory spinning around the sphere [3], leads to a maximally supersymmetric homogeneous wave background in $D=10$ dimensions, with metric

$$
d s^{2}=2 d u d v+\sum_{i} d x_{i}^{2}-\frac{1}{4} \mu^{2}\left(\sum_{i} x_{i}^{2}\right) d u^{2}
$$

where the sum runs from $i=1$ to 8 , together with a null Ramond flux $H=$

$\mu d u \wedge\left(d x^{1234}+d x^{5678}\right)$ [4. Homogeneous wave backgrounds in other dimensions $D$ or supported by different type of fluxes arise from other near-horizon geometries as discussed below. This supersymmetric background is amenable to exact quantization despite the existence of Ramond fluxes [5]. The corresponding limit from the dual gauge theory perspective was subsequently found, and a subset of the bulk string theory states was identified with non-chiral gauge invariant operators in a very pictorial way [6]. Most strikingly, the bulk dispersion relation was matched to the anomalous dimension in the gauge theory side by resumming an infinite number of non-planar diagrams.

While this scaling limit in effect gives a holographic description of these maximally supersymmetric plane wave backgrounds, it is legitimate to wonder if the large $J$ - fixed $(\Delta-J) S U(N)$ gluons are the most appropriate holographic degrees of freedom for this purpose. In particular, the only manifest symmetries in this description are the $S O(4) \times S O(4)$ (for $p=5$ ) unbroken symmetries, whereas the background exhibits a much larger symmetry algebra, namely the Wigner contraction of the original $S O(2,4) \times S O(6)$ symmetry (plus fermionic generators) [7]. Furthermore, the scaling limit provides additional discrete symmetries (such as the exchange of the 4 transverse directions in the $A d S_{5}$ part with the ones in $S^{5}$ ) which are completely obscure in this picture. Finally, given the stringy character of the relevant gauge theory excitations, it is rather tempting to fantasize the existence of an exotic string theory that would be holographically dual to the Green-Schwarz string in the bulk. Another hint that this may be so comes from the fact that the Penrose limit for a particle in the near-horizon geometry of a stack of D3-branes amounts to an infinite 
boost of the D3-branes themselves, T-dual to the near-critical electric field limit that gives rise to non-commutative open strings [8].

Rather than speculating further along these lines, it may be more enlightening to consider the pp-wave background in its own sake, and let the geometry decide what the holographic description is, if any. This approach is all the more useful as very similar geometries (but with different fluxes) arise in many different systems than the near horizon limit of D3-branes. Firstly, as already pointed out in [6], the near horizon limit of either M2-branes or M5-branes yields the maximally supersymmetric wave in 11 dimensions [9]. M-theory seems to be better behaved in this exact background, in particular the flat directions of the Matrix theory potential are lifted [6]. It would be interesting to make progress in quantizing membranes in these backgrounds with null curvature, but this lies outside the scope of this work (see [10] for a causally disconnected construction of instantons interpolating between different vacua in this model).

Secondly, the maximally supersymmetric $D=10$ type IIB wave background also arises in the Penrose limit of systems with less supersymmetry, such as $A d S_{5} \times T_{1,1}$, pointing to the existence of a subsector with $N=4$ supersymmetry in the dual $N=1$ gauge theory [11]. Next, as already pointed out in [6], the $D=6$ case arises in the Penrose limit of $A d S_{3} \times S^{3}$, and can be supported by either Ramond or Neveu-Schwarz flux $H=\mu d u \wedge\left(d x^{12}+d x^{34}\right)$, or by a combination of the two. The $D=4$ case, which will be the main focus of this work, arises from the Penrose limit of the flat NS5-brane near horizon geometry $\mathbb{R}^{1,5} \times \mathbb{R}_{\phi} \times S^{3}$ [14], by choosing a geodesic spinning along an equator of the sphere ${ }^{1}$ (it is worth noting that a geodesic along the radial direction supporting the linear dilaton yields a purely dilatonic wave, $\phi=\phi(u)$ with flat geometry, arguably the simplest of all plane wave backgrounds in string theory). It is supported by a Neveu-Schwarz flux $H=\mu d u \wedge d x^{1} \wedge d x^{2}$. The same $D=4$ geometry is obtained in the Penrose limit of the near horizon geometry of the NS5-brane wrapped on $S^{2}$ [12], giving another example of supersymmetry enhancement in the Penrose limit. Limits of many other backgrounds have been considered recently [13].

String theory propagation in similar gravitational wave backgrounds has been studied previously [15, 16, 17, 19, 20, 21]. In fact, the $D=4$ case is none other than the Nappi-Witten pp-wave background, i.e. the Wess-Zumino-Witten model on the centrally extended Euclidean group in two dimensions $E_{2}^{c}$ [15. Upon compactifying $u+v$, this model can be viewed as an exact background with constant magnetic field originating from the closed string sector, including the gravitational back-reaction [24]. Even with non-compact $u+v$, it shares many similarities with the Landau problem of charged particles in constant magnetic field, with the electric charge being identified with the light-cone momentum.

\footnotetext{
${ }^{1}$ This was independently noted in [12].
} 
From the algebraic point of view, the solvable group $E_{2}^{c}$ can also be viewed as the Heisenberg algebra $[P, Q]=K$ extended by the generator $J$ rotating position and momentum, $[J, P]=Q,[J, Q]=-P$; we will denote this algebra by $H_{4}$ in the sequel. The WZW model on this non-semi-simple group can also be obtained as a limit of the WZW model on $U(1)_{k} \times S U(2)_{k^{\prime}}$ where $k$ and $k^{\prime}$ are scaled to $+\infty$ and $-\infty$, respectively, with a fixed ratio [33]. In particular, its central charge is that of flat space, in agreement with the fact that there are no non-vanishing curvature invariants in this geometry. It was shown that the string theory on this background is exactly solvable both in a covariant gauge [17] and the light-cone gauge [24, 32, 22] and that it preserves 16 supercharges [19], just as the original NS5-brane system.

The Nappi-Witten background admits an obvious generalization in arbitrary dimension, by considering the WZW model on the extended Heisenberg group $H_{n}$ [16]. This yields the pp-wave background above for $D=2 n+2$, supported by a null Neveu-Schwarz flux $H=\mu d u \wedge \omega$ where $\omega$ is a symplectic form on the transverse space $\mathbb{R}^{D-2}$. One therefore has an exact conformal field theory description for all maximally symmetric Cahen-Wallach spaces (see [3] for a review of maximally symmetric Lorentzian spaces). As for all WZW models, these string backgrounds are exactly solvable, and they can be mapped to free fields as spelled out in [17, 19].

Motivated by their ubiquitous appearance in Penrose limits of near-horizon geometry, we will discuss general aspects of string theory and holography on homogeneous wave backgrounds supported by a null Neveu-Schwarz flux, setting $D=4$ in order to avoid cluttering the notation. We expect that as far as holography goes, these models share universal features with the ones supported by Ramond flux, of more direct interest in the AdS context (however we will point out specific differences in due time). In addition, these models are interesting laboratories to study string propagation in time-dependent or cosmological backgrounds.

Let us outline the structure of this paper and summarize our main conclusions. In section 2, we discuss general features of the wave background (1.1), and derive the dispersion relation in the tree-level approximation. In section 3 , we discuss the quantization of the RNS string in homogeneous wave backgrounds supported by Neveu-Schwarz flux, putting the results of [17, 19] in perspective. In particular we give a physical discussion of the "long string" states required by the spectral flow introduced in [17]. Moreover, discrete symmetries analogous to the $Z_{2}$ symmetry $A d S_{5} \leftrightarrow S^{5}$ arise here as well and we show that they are symmetries of the quantum theory. Section 2 and 3 can be read independantly of section 4 .

In section 4, we set up a bulk-to-boundary holographic correspondence for homogeneous wave backgrounds, irrespective of the dimension and flux. We argue that holography is most naturally implemented in the Poincaré patch (4.3), i.e. the maximal patch of the global geometry which is conformal to flat Minkowski space. Under this assumption, we find that the holographic direction is the light-cone coordinate $u$ (or equivalently $x^{+}$), and that the holographic dual lives on a $D-1$ dimensional holo- 
graphic screen ${ }^{2}$ at fixed light-cone time $u$. Its induced metric is therefore degenerate along the $v$ direction. This is in contrast to the AdS [25] and dS [26] cases, where the holographic dual lives on a time-like (resp. space-like) Cauchy surface, and rather analogous to Minkowski space [27]. As in de Sitter, the boundary off-shell correlators are identified with on-shell transition amplitudes between two screens at $x^{+}= \pm \infty$. Thus, we expect the study of holography in pp-wave backgrounds to shed light both on the problems of holography in Minkowski space-time, and in de Sitter. We determine the realization of the bulk isometries on the boundary, and propose that the holographic description is given by a new type of (possibly non-local) field theory with symmetry group $H_{D}^{L} \times H_{D}^{R}$ acting as displayed in (4.27), where $H_{D}$ is the Heisenberg algebra which defines the pp-wave background. In the appendices, we work out the Ward identities of two and three point functions in (4.28) and (D.17), give a sample computation for a self-interacting scalar field in the bulk, and express the result also in the harmonic oscillator basis.

A distinctive feature of this null holographic set-up is that, in contrast to the AdS and dS cases, the bulk equations of motion are first order in derivative of the holographic coordinate. While in AdS non-normalizable (resp. normalizable) solutions are in correspondence with boundary operators (resp. vacua), here only the analogue of the non-normalizable modes subsist. The vacuum structure therefore seems to be lost. As usual in light cone quantization the details of the vacuum are hidden. In order to have a well-defined boundary problem it is necessary to introduce an additional null holographic screen at fixed (infinite) $v$. The boundary values on this screen characterize the vacuum structure of the theory on the holographic screen at fixed $u$. Only trivial boundary conditions at $v \rightarrow-\infty$ leave the bulk symmetry unbroken.

\section{Geometry of homogeneous wave backgrounds}

\subsection{The homogeneous wave as a group manifold}

As we explained in the introduction, the homogeneous plane wave metric (1.1), supported by a Neveu-Schwarz flux, can be constructed as the manifold of the $D-1$ dimensional Heisenberg group, extended by a generator rotating the positions and momenta:

$$
H_{D}: \quad\left[P_{i}, P_{j}\right]=\omega_{i j} K, \quad\left[J, P_{i}\right]=\omega_{i j} P_{k}
$$

where $K$ is a central element. This can also be thought as the non-commutative phase space for a particle in $D-2$ dimensions in a constant magnetic field $B=\omega_{i j} d x^{i j}$, with $J$ generating a rotation in each of the proper planes of $B$. The center of the group is generated by $K$ and the Casimir $C=P_{T}^{2}+2 J K$ where $P_{T}^{2}=\sum P_{i}^{2}$. Restricting

\footnotetext{
${ }^{2}$ This diverges from the work in [36] that appeared as the present work was prepared for publication, and that advocates an Euclidean $(D-2) / 2$-dimensional dual space.
} 
for simplicity to $D=4$, and letting $\omega_{12}=1$, the group has the (non-unitary) matrix representation

$$
g\left(u, v, a_{1}, a_{2}\right)=e^{a_{1} P_{1}+a_{2} P_{2}} e^{u J+v K}=\left(\begin{array}{cccc}
1 & a_{1} \sin u-a_{2} \cos u & a_{1} \cos u+a_{2} \sin u & 2 v \\
0 & \cos u & -\sin u & a_{1} \\
0 & \sin u & \cos u & a_{2} \\
0 & 0 & 0 & 1
\end{array}\right)
$$

In this presentation the generator $J$ appears to be compact, while the other ones are non-compact. We will consider mostly the universal cover in order to avoid closed light-like curves. However for some DLCQ applications it may be desirable to keep $u$ compact. We will argue later that, for compact $u$, string theory is only consistent if $u$ has $2 \pi / n$ periodicity (after setting the mass scale of the background to 1 ).

A bi-invariant metric can be obtained as $d s^{2}=\left\langle g^{-1} d g, g^{-1} d g\right\rangle$, where $\langle\cdot, \cdot\rangle$ is an invariant symmetric form on the Lie algebra. The trace in the above matrix representation yields a degenerate metric $\operatorname{Tr}\left(g^{-1} d g g^{-1} d g\right)=-2 d u^{2}$, however there is another invariant form, $\left\langle P_{i}, P_{j}\right\rangle=\delta_{i j},\langle J, K\rangle=1$ with other components vanishing. This yields a non-degenerate metric,

$$
d s^{2}=2 d u d v+d a_{1}^{2}+d a_{2}^{2}+\left(a_{2} d a_{1}-a_{1} d a_{2}\right) d u
$$

This is supported by the Neveu-Schwarz flux $H=d u \wedge d a_{12}$. In this form, one recognizes a constant magnetic background $F=d a_{12}$ for the Kaluza-Klein gauge field $g_{\mu u}$ - except for the fact that the $u$ coordinate is null. The Laplacian in these "magnetic" coordinates reads

$$
\Delta=2 \partial_{u} \partial_{v}+\left(\partial_{1}-\frac{1}{2} a_{2} \partial_{v}\right)^{2}+\left(\partial_{2}+\frac{1}{2} a_{1} \partial_{v}\right)^{2}
$$

so that the free wave equation $\left(\Delta-m^{2}\right) \phi=0$ reduces to the Schrodinger equation for a particle of charge $p_{+}$(the momentum along $v$ ) in a constant magnetic field. Changing to the rotating frame at the Larmor frequency, $a_{1}+i a_{2}=e^{i u / 2}\left(x_{1}+i x_{2}\right)$, leads ${ }^{3}$ to the more familiar metric

$$
d s^{2}=2 d u d v+d x_{1}^{2}+d x_{2}^{2}-\frac{1}{4}\left(x_{1}^{2}+x_{2}^{2}\right) d u^{2}+d s_{\perp}^{2},
$$

supported by the Neveu-Schwarz flux $H=d u \wedge d x^{12}$. The equations of motion are easily seen to be satisfied, since the only non-vanishing component of the Ricci tensor, $R_{u u}=1 / 2$, is compensated by the NS flux $H_{u i j} H_{u}^{i j}$.

\footnotetext{
${ }^{3}$ Changing to the frame rotating at twice the Larmor frequency, $a_{1}+i a_{2}=e^{i u}\left(b_{1}+i b_{2}\right)$, leads back to a magnetic background but with opposite magnetic field. It amounts to passing $e^{a_{i} P_{i}}$ through $e^{u J+v K}$ in (2.2).
} 


\subsection{Isometries and geodesics}

The metric (2.4) or (2.5) is by construction invariant under $H_{4 L} \times H_{4 R}$ acting from the left and the right on the group $H_{4}$ itself, respectively. The group law is easily computed, e.g. using the Baker-Campbell-Hausdorff formula [35], or using the matrix representation,

$g\left(u_{1}, v_{1}, z_{1}\right) g\left(u_{2}, v_{2}, z_{2}\right)=g\left(u_{1}+u_{2}, v_{1}+v_{2}-\frac{1}{2} \Im\left(z_{1} e^{-i\left(u_{1}+u_{2}\right) / 2} \bar{z}_{2}\right), z_{1} e^{-i u_{2} / 2}+z_{2} e^{i u_{1} / 2}\right)$

in particular the inverse is $g(u, v, z)^{-1}=g(-u,-v,-z)$. Since the element $K$ is central, the resulting group of isometries is seven-dimensional. The Killing vectors associated to the left and right action follow ${ }^{4}$ from the group law, and read:

$$
\begin{gathered}
K=\partial_{v} \\
J_{L}=\partial_{u}+\left(x_{1} \partial_{2}-x_{2} \partial_{1}\right) / 2 \\
J_{R}=\partial_{u}-\left(x_{1} \partial_{2}-x_{2} \partial_{1}\right) / 2 \\
P_{1 L}=\frac{1}{2}\left(x_{2} \cos \frac{u}{2}+x_{1} \sin \frac{u}{2}\right) \partial_{v}+\cos \frac{u}{2} \partial_{1}-\sin \frac{u}{2} \partial_{2} \\
P_{1 R}=\frac{1}{2}\left(-x_{2} \cos \frac{u}{2}+x_{1} \sin \frac{u}{2}\right) \partial_{v}+\cos \frac{u}{2} \partial_{1}+\sin \frac{u}{2} \partial_{2} \\
P_{2 L}=\frac{1}{2}\left(-x_{1} \cos \frac{u}{2}+x_{2} \sin \frac{u}{2}\right) \partial_{v}+\sin \frac{u}{2} \partial_{1}+\cos \frac{u}{2} \partial_{2} \\
P_{2 R}=\frac{1}{2}\left(x_{1} \cos \frac{u}{2}+x_{2} \sin \frac{u}{2}\right) \partial_{v}-\sin \frac{u}{2} \partial_{1}+\cos \frac{u}{2} \partial_{2}
\end{gathered}
$$

where $\partial_{i}:=\partial / \partial x_{i}$. In particular, $K, J_{L}$ and $J_{R}$ are null Killing vectors. The combination $J_{L}+J_{R}=2 \partial_{u}$ is a timelike Killing vector, and will play a special role in our discussion as the holographic generator.

\subsection{Geodesics and waves}

It is of interest to investigate the free trajectories in this background. This is especially simple in this homogeneous case, since the geodesic flow is generated by the left (or right) action of a one-parameter subgroup of $H_{4}, g(\tau)=\exp \left(2 \tau\left(p_{+} J+p_{-} K+\right.\right.$ $\left.\left.p_{1} P_{1}+p_{2} P_{2}\right)\right)$ depending on the initial momentum. Starting without loss of generality from the origin $(u, v, z)=(0,0,0)$ of the group, one finds that the geodesics are parameterized by

$$
\begin{aligned}
& u(\tau)=2 p_{+} \tau \\
& v(\tau)=\left(2 p_{-}+\frac{p_{1}^{2}+p_{2}^{2}}{p_{+}}\right) \tau-\frac{\sin 2 p_{+} \tau}{2 p_{+}^{2}}\left(p_{1}^{2}+p_{2}^{2}\right) \\
& z(\tau)=2 \sin \left(p_{+} \tau\right)\left(p_{1}+i p_{2}\right) / p_{+}
\end{aligned}
$$

\footnotetext{
${ }^{4}$ In our conventions, the right generators have opposite commutation relations to the left ones.
} 
The particle therefore oscillates in the harmonic potential well in the transverse $z$ direction with a period $\Delta \tau=2 \pi / p_{+}$(the period is $\pi / p_{+}$in $w$ coordinates). During one period, the particle moves linearly by $\Delta u=4 \pi$ in the $u$ direction, and by $\Delta v=4 \pi p^{2} / 2 p_{+}^{2}$ in the $v$ direction, where $p^{2}=2 p_{+} p_{-}+p_{1}^{2}+p_{2}^{2}$ is the invariant momentum square at $\tau=0$. The mean velocity $\Delta v / \Delta u=p^{2} / 2 p_{+}^{2}$ therefore depends on the type of trajectory under consideration: massive particles have a net forward motion along the $v$ axis (as $u$ increases) while space-like trajectories go backward in $v$. Massless particles on the other hand are confined in the $v$ direction on an interval of length $\left(p_{1}^{2}+p_{2}^{2}\right) / p_{+}^{2}$. In either case, the Zitterbewegung-like motion along $v$ forbids to take it as a time coordinate, in contrast to $u$ which is a single valued function of the proper time $\tau$. If on the other hand the light-cone momentum $p_{+}$vanishes, the potential well disappears, and the motion is linear in the $\left(v, x_{1}, x_{2}\right)$ plane with fixed $u$. This in general corresponds to a space-like geodesic. Null geodesics with $p_{+}=0$ must also have $p_{1}=p_{2}=0$, and correspond to massless particles traveling parallel to the wave. Starting away from the bottom of the potential changes the details of the motion in the transverse plane, but the discussion of the motion in the $(u, v)$ directions still applies.

Having at hand the general form of the trajectories, one readily obtains the geodesic (Lorentzian) distance $r$ between any two points,

$r^{2}=2\left(u-u^{\prime}\right)\left(v-v^{\prime}\right)+\frac{u-u^{\prime}}{2 \sin \left(\frac{u-u^{\prime}}{2}\right)}\left[\left(x_{1}^{2}+x_{2}^{2}+x_{1}^{\prime 2}+x_{2}^{\prime 2}\right) \cos \left(\frac{u-u^{\prime}}{2}\right)-2\left(x_{1} x_{1}^{\prime}+x_{2} x_{2}^{\prime}\right)\right]$

The geodesic distance therefore goes to infinity when $u-u^{\prime}=2 \pi n, n \in \mathbb{Z}$, unless $\left(x_{1}, x_{2}\right)=\left(x_{1}^{\prime}, x_{2}^{\prime}\right)$ for $n$ even, or $\left(x_{1}, x_{2}\right)=\left(-x_{1}^{\prime},-x_{2}^{\prime}\right)$ for $n$ odd. This agrees with the periodicity of the trajectories in the transverse plane.

These features of the classical trajectories carry over to the propagation of waves in this background. Scalar waves satisfy $\left(\Delta-m^{2}\right) \phi=0$ where the Laplace operator can be computed either from the metric (1.1) or from the Casimir of the left or right generators,

$$
\Delta=2 \partial_{u} \partial_{v}+\frac{1}{4}\left(x_{1}^{2}+x_{2}^{2}\right) \partial_{v}^{2}+\left(\partial_{1}^{2}+\partial_{2}^{2}\right)+\Delta_{\perp}
$$

Acting on a plane wave $e^{i\left(u p_{-}+v p_{+}\right)}$with non-zero $p_{+}$, one gets the Hamiltonian for a harmonic oscillator in two dimensions with frequency $\left|p_{+}\right| / 2$. Its ground state is the Gaussian wave function

$$
\phi(u, v, z)=\exp \left[i\left(p_{+} v+p_{-} u+p_{\perp} x_{\perp}\right)-\frac{1}{4}\left|p_{+}\right| z \bar{z}\right]
$$

while arbitrary states are obtained by acting $n_{L}$ times (resp. $n_{R}$ ) with the creation operators $P_{L}^{-}$and $P_{R}^{+}$,

$$
\begin{array}{ll}
P_{L}^{+}=e^{i u / 2}\left(4 \partial_{\bar{z}}-i z \partial_{v}\right), & P_{L}^{-}=e^{-i u / 2}\left(4 \partial_{z}+i \bar{z} \partial_{v}\right) \\
P_{R}^{+}=e^{-i u / 2}\left(4 \partial_{\bar{z}}+i z \partial_{v}\right), & P_{R}^{-}=e^{i u / 2}\left(4 \partial_{z}-i \bar{z} \partial_{v}\right)
\end{array}
$$


where $P^{ \pm}=P_{1} \pm i P_{2}, z=x_{1}+i x_{2}, \partial_{z}=\left(\partial_{1}-i \partial_{2}\right) / 2$. The dispersion relation is easily seen to be

$$
2 p_{+} p_{-}+\left|p_{+}\right|\left(n_{L}+n_{R}+1\right)+p_{\perp}^{2}+m^{2}=0
$$

while the helicity in transverse place is $h=\operatorname{sgn}\left(p_{+}\right)\left(n_{L}-n_{R}\right)$. We will re-derive this spectrum more systematically in the next section. For $p_{+}=0$, the wave equation reduces to the Laplace operator in transverse space, and has no solution for positive $m^{2}$. For $m^{2}=0$, the solution is an arbitrary profile in $u$, independent of the remaining coordinates. It is interesting to note from the dispersion relation (2.13) that massless states can correspond to either $p_{+}=0$ and arbitrary $p_{-}$, or to $p_{-}=\operatorname{sgn}\left(p_{+}\right)\left(n_{L}+\right.$ $\left.n_{R}+1 / 2\right)$ and arbitrary $p_{-}$(with $p_{\perp}=0$ of course).

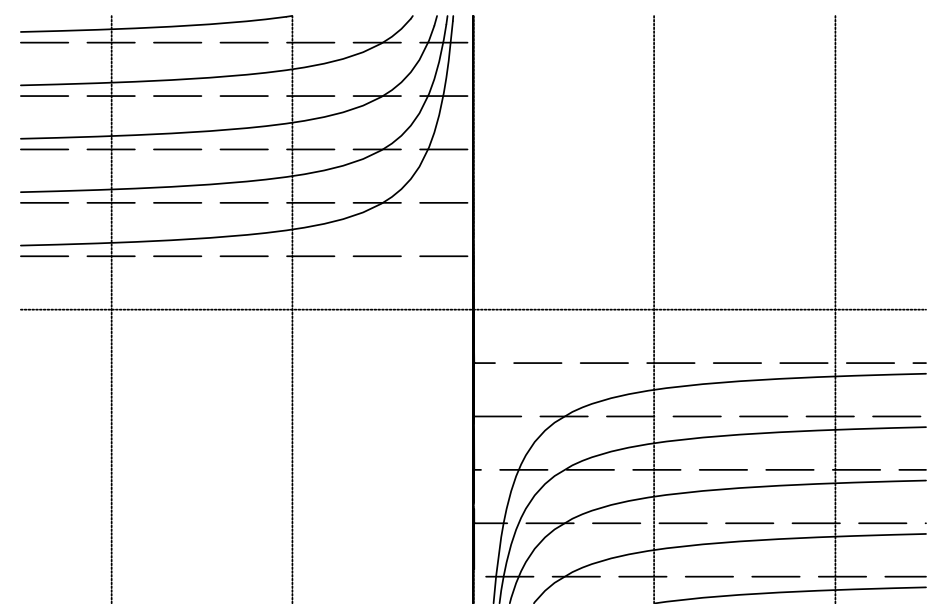

Figure 1: Dispersion relation in the $\left(p_{+}, p_{-}\right)$plane.

For zero mass (in dotted lines), the spectrum of $p_{-}$takes discrete positive values (resp. negative) at $p_{+}<0$ (resp. $p_{+}>0$ ), and forms a lowest (resp. highest) weight representation of $H_{4 L} \times H_{4 R}$. At $p_{+}=0$, the spectrum of $p_{-}$is continuous, with each value transforming as a singlet. For $m^{2}>0,\left|p_{-}\right|$develops a mass gap, and there are no states at $p_{+}=0$ anymore. Vertical lines denote integer $p_{+}$. For the bosonic string, the spectrum is further tilted and there is a tachyonic state.

\subsection{Representation theory of the extended Heisenberg group $H_{D}$}

The spectrum found above should fall into unitary irreducible representations of the left and right $H_{4}$ actions. The unitary irreps of $H_{4}$ were constructed in [28] and from a more applied point of view in [17]. We will briefly recall their construction, in notations consistent with this paper (in particular all generators $P_{1,2}, K, J$ are taken to be anti-hermitian).

The unitary irreps can be constructed by diagonalizing simultaneously the commuting operators $C, K, J$ :

$$
J|j, t\rangle=i j|j, t\rangle, \quad K|j, t\rangle=i t|j, t\rangle, \quad C|j, t\rangle=C|j, t\rangle
$$


with $j$ and $t$ real. $K$ and $C$ are constant in a given irreducible representation. Since $P_{1}^{2}+P_{2}^{2}$ is negative definite, we must have $C+2 j t \leq 0$. The operators $P^{ \pm}=$ $P_{1} \pm i P_{2}$ can be thought of as raising and lowering operators for $J$, as $\left[J, P^{ \pm}\right]=\mp i P^{ \pm}$. Choosing an orthonormal basis and making a choice of phases, they act as

$$
P^{ \pm}|j, t\rangle=\sqrt{-[C+t(2 j \mp 1)]}|j \mp 1, t\rangle
$$

We then have the following representations:

(I) Representations without highest nor lowest weight have $j$ ranging over $j_{0}+\mathbb{Z}$, and $j_{0}$ can be chosen in $[0,1$ [ without loss of generality. In order that $C+2 j t \leq 0$ for all states, we must have $t=0$ and $C<0$.

(II) Representations with lowest weight $P^{-}|j, t\rangle=0$ have $C=-t(2 j+1)$. The negativity of $P_{1}^{2}+P_{2}^{2}$ implies $t>0$. The spectrum of $J$ is bounded from above, $[J]=j-\mathbb{N}$. The Casimir $C$ is positive for $j<-1 / 2$.

(III) Representations with highest weight $P^{+}|j, t\rangle=0$ have $C=-t(2 j-1)$. The negativity of $P_{1}^{2}+P_{2}^{2}$ implies $t<0$. The spectrum of $J$ is bounded from below, $[J]=j+\mathbb{N}$. The Casimir $C$ is positive for $j>1 / 2$.

(IV) Representations with both highest weight $j$ and lowest weight $j^{\prime}$ must have $j=j^{\prime}$ : there are therefore one-dimensional only. They have $C=t=0$, and $j$ is any real number.

The spectrum of the lowest and highest weight representations II and III can be summarized by $C=-2 t j-|t|$ and $J=j-\operatorname{sgn}(t) \mathbb{N}$. For a fixed value of the charges $t$ and $J$, the Casimir is then

$$
C=-2 t J-(2 \mathbb{N}+1)|t|
$$

In particular, irrespective of the sign of $t$, it is always discrete and, for a fixed $J$, bounded from above. As $t$ goes to zero, the Casimir goes to zero. For $t=0$ strictly, we have a singlet at $C=0$ (type IV), and a continuum of states with $C<0$ (type i).

Let us now go back to the spectrum of the Laplacian in the background (1.1), and fit it into representations of $H_{4 L} \times H_{4 R}$. The eigenvalues of $K, J_{L}, J_{R}$ are identified as $t_{L}=t_{R}=p_{+}, j_{L}=p_{-}-h / 2, j_{L}=p_{-}+h / 2$, while the Casimir $C=C_{L}=$ $C_{R}$ is identified with the mass squared. For representations with highest or lowest weight on both sides, (2.16) implies $j_{L}=j_{R}$ on the highest (lowest weight), which should therefore have zero helicity. If $u$ was taken to be compact, this would further imply that $j_{L}+j_{R}=2 p_{-}$is quantized. The sum of (2.16) on the left and right side reproduces the dispersion formula (2.13), while the difference identifies $h=$ $\epsilon\left(p_{+}\right)\left(n_{L}-n_{R}\right)$. 
The spectrum of the light-cone Hamiltonian $p_{-}$as a function of the light-cone momentum $p_{+}$for a fixed value of $m^{2}$ (or more accurately $m^{2}+p_{\perp}^{2}$ ) is represented in Figure 1. For $m^{2}=0$, we have a highest weight representation of type II at $p_{+}<0$, a lowest weight representation of type III at $p_{+}>0$, and an infinity of type IV singlet representations at $p_{+}=0$, arbitrary $p_{-}$. The light-cone energy of the type II and III representations is independent of the momentum. For $m^{2}>0$, we have only type II and III representations at $p_{+}>0$ and $p_{+}<0$, respectively. In contrast to flat space, the light-cone Hamiltonian has a mass gap, $\left|p_{-}\right|>1 / 2$. Representations of type IV exist only for tachyonic mass, and will not occur in the superstring case.

\section{Strings in Neveu-Schwarz homogeneous pp-waves}

String theory in the Nappi-Witten background (or its higher dimensional generalizations) can be solved in the light-cone gauge, where the transverse scalars become free massive fields [24, 32]. Alternatively, one may avoid the light-cone gauge and make use instead of the $H_{D L} \times H_{D R}$ current algebra

$$
J_{a}(z) J_{b}\left(z^{\prime}\right)=\frac{G_{a b}}{\left(z-z^{\prime}\right)^{2}}+f_{a b}{ }^{c} \frac{J_{c}\left(z^{\prime}\right)}{\left(z-z^{\prime}\right)}+\text { regular }
$$

where $G_{a b}$ is the non-degenerate invariant symmetric form on the Lie algebra introduced before, $\left\langle P_{1}, P_{2}\right\rangle=\langle J, K\rangle=1$, rescaled by a factor of the affine level $k$.

The analogue of the affine-Sugawara stress tensor, satisfying the Master equation [29], is then given by $T=-L^{a b} J_{a} J_{b}$ where $L^{a b}=G^{a b} / 2+\tilde{G}^{a b} /\left(2 k^{2}\right)$, where $\tilde{G}^{a b}$ is the degenerate invariant symmetric form with only non-vanishing element $\tilde{G}^{K K}=1$. The affine level $k$ can be set to one by rescaling $\alpha^{\prime}$.

The left-moving and right-moving currents can be computed from $J_{L}(z)=$ $\left\langle g^{-1} \partial g, J\right\rangle, J_{R}(z)=\left\langle\bar{\partial} g g^{-1}, J\right\rangle$, respectively. In the coordinate system (2.2), we find that the left-moving currents take a simple expression,

$$
\begin{aligned}
K_{L}=\partial u, & J_{L}=\partial v+\frac{1}{2}\left(a_{2} \partial a_{1}-a_{1} \partial a_{2}\right), \\
P_{L}^{+}=e^{-i u} \partial w, & P_{L}^{-}=e^{i u} \partial \bar{w},
\end{aligned}
$$

whereas the right-moving currents are more awkward,

$$
\begin{aligned}
K_{R}=\bar{\partial} u, & J_{R}=\bar{\partial} v+\frac{1}{2}\left(a_{2} \bar{\partial} a_{1}-a_{1} \bar{\partial} a_{2}\right)-\frac{1}{2}\left(a_{1}^{2}+a_{2}^{2}\right) \bar{\partial} u \\
P_{R}^{+}=\bar{\partial} w-i w \bar{\partial} u, & P_{R}^{-}=\bar{\partial} \bar{w}+i \bar{w} \bar{\partial} u
\end{aligned}
$$

Changing to the rotating frame at twice the Larmor frequency would reverse the situation. In fact, it was shown in [17] that the current algebra has the free boson representation, reminiscent of (3.2),

$$
K=\partial u, \quad J=\partial v, \quad P_{L}^{+}=e^{-i u} \partial w, \quad P_{L}^{-}=e^{i u} \partial \bar{w},
$$


where $P_{L}^{ \pm}=\left(P_{1} \pm i P_{2}\right) / \sqrt{2}$. The scalars $(u, v, w, \bar{w})$ have free boson propagators,

$$
\left\langle x^{\mu}(z) x^{\nu}\left(z^{\prime}\right)\right\rangle=\eta^{\mu \nu} \log \left(z-z^{\prime}\right)
$$

with metric $\eta_{\mu \nu} d x^{\mu} d x^{\nu}=2 d u d v+2 d w d \bar{w}$.

This free-field representation amounts to choosing different coordinate systems for the left and right movers, namely $w=a_{1}+i a_{2}$ on the left and $w=b_{1}+i b_{2}$ on the right, with $e^{-i u / 2}\left(a_{1}+i a_{2}\right)=x_{1}+i x_{2}=e^{i u / 2}\left(b_{1}+i b_{2}\right)$. In addition, one drops the singular helicity operator in $J(z)$. One may then check that the operators (3.6) satisfy the current algebra (3.1). The stress tensor then takes the free boson form, $T=-\frac{1}{2} \eta_{\mu \nu} \partial x^{\mu} \partial x^{\nu}$.

With these notations, the vertex operator $: e^{i p_{-} u+i p_{+} v}:$ generates the state $\left|p_{+}, p_{-}\right\rangle$with $t=p_{+}, j=p_{-}$and $L_{0}=-p_{+} p_{-}$. This free-field resolution of the algebra generalizes in an obvious fashion to all Cahen-Wallach (CW) spaces with NS-NS two-form, by adding extra pairs of raising and lowering operators $P_{L i}^{ \pm}$expressed in terms of free complex coordinates.

\subsection{Chiral primaries and vertex operators}

As shown in [17] type I representations correspond to the states $\left|p_{+}, p_{-}\right\rangle$with $p_{+}=0$ and all oscillator states generated by $P^{ \pm}$. We should note that such states are invisible in the light-cone gauge. It turns out that only tachyonic or massless states may belong to this class. In particular, for the superstring, where tachyons are absent, the only physical states in this class are the ground-states $\left|p_{+}=0, p_{-}\right\rangle \otimes|0\rangle$ where $|0\rangle$ is the vacuum of the extra six-dimensional CFT. They correspond to the massless modes traveling with the wave. Their tree level interactions are the same as in Minkowski space.

Type II primaries can be represented as 17

$$
R_{p_{+}, p_{-}}^{I I}=\exp \left[i\left(p_{-} u+p_{+} v\right)\right] H_{p_{+}}\left(x^{1}, x^{2}\right) .
$$

Here we have $p_{+}>0$. The highest weight condition implies that

$$
\partial w(z) H_{p_{+}}\left(z^{\prime}\right) \sim\left(z-z^{\prime}\right)^{-1-p_{+}}, \quad \partial \bar{w}(z) H_{p_{+}}\left(z^{\prime}\right) \sim\left(z-z^{\prime}\right)^{p_{+}},
$$

It is apparent from the OPEs above that the vertex operator $V_{p_{+}}^{I I}$ is an operator that twists the transverse plane coordinates via the transformation

$$
w\left(e^{2 \pi i} z\right)=e^{-2 \pi i p_{+}} w(z), \bar{w}\left(e^{2 \pi i} z\right)=e^{2 \pi i p_{+}} \bar{w}(z) .
$$

Equivalently, they modify the periodicity of the free boson $w$ along the world-sheet

spatial coordinate [17, 19, 24, 32]. Note that in general the twist need not be a fractional number. The conformal weight of this twist field is $p_{+}\left(1-p_{+}\right) / 2$ so that the total $L_{0}$ conformal weight is

$$
\Delta=p_{+} p_{-}+\frac{1}{2} p_{+}\left(1-p_{+}\right), \quad p_{+}>0
$$


in agreement with the Casimir value of the classical $H_{4}$ algebra, up to the stringy correction term quadratic in $p_{+}$. These states correspond to the confined Landautype harmonic oscillator states in the transverse plane (they can of course carry momentum in the extra $10-D$ directions). The energy formula (3.11) is only valid for $0 \leq p_{+}<1$, since as $p_{+} \rightarrow p_{+}+1$ the free boson $w$ is twisted by the same amount $e^{-2 \pi i p_{+}}$.

Let us expand the free boson $\partial w, \partial \bar{w}$ in oscillator modes $a_{k+p_{+}}$and $\bar{a}_{k-p_{+}}$. The action of the affine generators $P_{n}^{ \pm}$can then be written as

$$
\begin{aligned}
& P_{k}^{+}\left|p_{+}, p_{-}\right\rangle \otimes\left|H_{p_{+}}\right\rangle=\left|p_{+}, p_{-}-1\right\rangle \otimes a_{k+p_{+}}\left|H_{p_{+}}\right\rangle \\
& P_{k}^{-}\left|p_{+}, p_{-}\right\rangle \otimes\left|H_{p_{+}}\right\rangle=\left|p_{+}, p_{-}+1\right\rangle \otimes \bar{a}_{k-p_{+}}\left|H_{p_{+}}\right\rangle
\end{aligned}
$$

Due to (3.9), the oscillators $a_{k+p_{+}}$for $k \geq 1$ and $\bar{a}_{k-p_{+}}$for $k \geq 0$ annihilate the twisted vacuum, while the other ones act as creation operators. A general state can be written as

$$
\prod_{k=0}^{\infty}\left(a_{-k+p_{+}}\right)^{N_{k}} \prod_{\bar{k}=1}^{\infty}\left(\bar{a}_{-\bar{k}-p_{+}}\right)^{\bar{N}_{\bar{k}}}\left|p_{+}, p_{-}-\sum N_{k}+\sum \bar{N}_{\bar{k}}\right\rangle \otimes\left|H_{p_{+}}\right\rangle
$$

Its conformal dimension is

$$
\begin{aligned}
L_{0}= & p_{+} P_{-}+\frac{1}{2} p_{+}\left(1-p_{+}\right)+\sum_{k=0}^{\infty}\left(k-p_{+}\right) N_{k}+\sum_{\bar{k}=1}^{\infty}\left(\bar{k}+p_{+}\right) N_{\bar{k}} \\
& =p_{+} P_{-}+p_{+} n+\frac{1}{2} p_{+}\left(1-p_{+}\right)+N
\end{aligned}
$$

where $N=\sum_{k=0}^{\infty} k N_{k}+\sum_{\bar{k}=1}^{\infty} \bar{k} N_{\bar{k}}$ is the total oscillation number, $n=\sum N_{k}-\sum \bar{N}_{\bar{k}}$ and $P_{-}=p_{-}-n$ is the light-cone energy of the resulting state. Type III primaries are conjugate to type II primaries. They are essentially constructed like the type II but starting on the $\mid p_{+}\left\langle 0, p_{-}\right\rangle$state [17]. They have the same conformal weights as in (3.11) but with $p_{+} \rightarrow\left|p_{+}\right|$.

\subsection{Spectral flow and long strings}

As we have seen, the primary field (3.8) only gives rise to states with $0 \leq p_{+}<1$. Increasing $p_{+}$by one amounts to performing the spectral flow

$$
\begin{aligned}
& J_{m} \rightarrow J_{m}, \quad K_{m} \rightarrow K_{m}+i w \delta_{m, 0}, \quad P_{m}^{ \pm} \rightarrow P_{m \pm w}^{ \pm} \\
& L_{0} \rightarrow L_{0}-i w\left(K_{0}-J_{0}\right)-\frac{1}{2} w(1-w) \delta_{m, 0}
\end{aligned}
$$

When $w \in \mathbb{Z}$ we obtain an isomorphic algebra, but highest weight vectors are now defined to be eigenmodes of different Cartan generators, $J_{0}$ and $K_{0}+i w$, and annihilated by operators $P_{n \pm w}^{ \pm}$with $n \geq 0$. One therefore obtains different representations, with conformal dimension

$$
\Delta(w)=\Delta(0)-w\left(p_{+}-p_{-}\right)+\frac{1}{2} w(1-w)=p_{+}^{\prime} p_{-}+\frac{1}{2} p_{+}^{\prime}\left(1-p_{+}^{\prime}\right)
$$


where $p_{+}^{\prime}=p_{+}+w$ is the new momentum. The formula (3.11) appears to still be valid for $p_{+}>0$ states outside the range $0<1<p_{+}$, but with the understanding that a representation of arbitrary $p_{+}>0$ is really obtained from the standard type II representation of light-cone momentum $p_{+} \bmod 1$ by flowing by $\left[p_{+}\right]$units. A similar statement applies to the $p_{+}<0$ states.

It is useful to understand the geometric meaning of these new representations, in particular in order to determine the allowed spectral flows. Starting from a classical solution $g(\tau, \sigma)=g(\tau+\sigma) g(\tau-\sigma)$ of the WZW model, the spectral flow by $w_{L}$ units on the left and $w_{R}$ on the right acts as

$$
g(\tau, \sigma) \rightarrow e^{w_{L}(\tau+\sigma) J} g(\tau, \sigma) e^{w_{R}(\tau-\sigma) J}
$$

In terms of the string embedding coordinates, this amounts to transforming

$$
\begin{aligned}
u & \rightarrow u+\left(w_{L}+w_{R}\right) \tau+\left(w_{L}-w_{R}\right) \sigma \\
v & \rightarrow v \\
x_{1}+i x_{2} & \rightarrow e^{\frac{i}{2}\left(w_{L}+w_{R}\right) \sigma+\frac{i}{2}\left(w_{L}-w_{R}\right) \tau}\left(x_{1}+i x_{2}\right)
\end{aligned}
$$

Starting from the generic particle trajectory $g(\tau)=e^{2 \tau\left(p_{-} J+p_{+} K+p_{i} P_{i}\right)}$ that we analyzed in (2.8), one obtains string worldsheets parameterized by

$$
\begin{aligned}
& u(\tau, \sigma)=\left(2 p_{+}+w_{L}+w_{R}\right) \tau+\left(w_{L}-w_{R}\right) \sigma \\
& v(\tau, \sigma)=\frac{2 p_{-} p_{+}+p_{1}^{2}+p_{2}^{2}}{p_{+}} \tau-\left[p_{1}^{2}+p_{2}^{2}\right] \frac{\sin \left(2 p_{+}\right)}{2 p_{+}^{2}} \\
& z(\tau, \sigma)=2 \sin \left(p_{+} \tau\right) e^{\frac{i}{2}\left(w_{L}+w_{R}\right) \sigma+\frac{i}{2}\left(w_{L}-w_{R}\right) \tau}\left[p_{1}+i p_{2}\right] / p_{+}
\end{aligned}
$$

Unless the coordinate $u$ is compact, one should therefore only allow for symmetric spectral flow $w_{L}=w_{R}:=w$. The resulting configuration is a string winding $w$ times around the bottom of the potential in transverse space. This state carries charges $P_{-}=p_{-}, P_{+}=p_{+}+w$, and has conformal dimension $L_{0}=\bar{L}_{0}=\frac{1}{2}\left(p_{1}^{2}+p_{2}^{2}\right)+\left(p_{+}+\right.$ $w) p_{-}$, to be equated to the conformal weight in the flat transverse directions $h$. The spectral flow from a time-like geodesic (resp. space-like) therefore yields a state of $p_{-}<h / w\left(\right.$ resp. $\left.p_{-}>h / w\right)$. The light-cone energy is extremized when $p_{i}=0$, where the string degenerates to a particle sitting at the bottom of the harmonic potential well. On the other hand, one may consider the spectral flow from a $p_{+}=0$ geodesic, with momentum $\left(0, p_{-}, p_{1}, p_{2}\right)$ and starting at an arbitrary position $\left(0,0, x_{1}, x_{2}\right)$ in transverse space. One obtains after a symmetric flow

$$
\begin{aligned}
& u(\tau, \sigma)=2 w \tau \\
& v(\tau, \sigma)=2 p_{-} \tau \\
& z(\tau, \sigma)=e^{i w \sigma}\left[\left(x_{1}+i x_{2}\right)+\tau\left(p_{1}+i p_{2}\right)\right]
\end{aligned}
$$


This state carries charge $J_{L}=J_{R}=w, K_{L}=p_{-}, K_{R}=p_{-}+p_{1} x_{2}-p_{2} x_{1}$, and has conformal dimensions $L_{0}=\frac{1}{2}\left(p_{1}^{2}+p_{2}^{2}\right)+p_{-} w, \bar{L}_{0}=L_{0}+w\left(p_{1} x_{2}-p_{2} x_{1}\right)$. The Virasoro conditions $L_{0}+\bar{L}_{0}=2 h, L_{0}-\bar{L}_{0}=0$ determine the momenta $p_{i}$ in terms of the initial position $x_{i}$. The resulting state

$$
z(\tau, \sigma)=e^{i w \sigma}\left(x_{1}+i x_{2}\right)\left[1 \pm 2 \tau \sqrt{\frac{2\left(h-p_{-} w\right)}{x_{1}^{2}+x_{2}^{2}}}\right]
$$

describes a closed string of winding number $w$ around the bottom of the potential, contracting or expanding linearly with time. At the critical value $p_{-}=h / w$, this becomes a static string, whose radius can stretch in the transverse directions at no cost in energy. Its light-cone momentum $p_{+}$is fixed to be integer. This string is the analogue of the long string appearing in Euclidean $A d S_{3}$ [37]. Upon compactifying $u$, one obtains for $w_{L} \neq w_{R}$ strings that wind around $u$ and have correlated angular momentum in the transverse direction. Note that the radius of $u$ is fixed to $2 \pi$, since $w_{L}-w_{R}$ is required to be integer. It would be interesting to relate these extended states to the thermal instabilities found in [30].

\subsection{String spectrum}

We can now describe the spectrum of physical states of string theory in the background $H_{4} \times C_{6}$ where $C_{6}$ stands for the extra six-dimensional background. We will assume that $C_{6}$ is the Euclidean space $\mathbb{R}^{6}$, as e.g. in the Penrose limit of the NS5-brane case. We choose a physical gauge ${ }^{5}$ by dropping the non-trivial oscillator modes of the currents $J_{L}, K_{L}, J_{R}, K_{R}$.

The level zero left- and right-moving generators are identified as $K_{L 0}=K_{R 0}=$ $p_{+}, J_{0 L}+J_{0 R}=2 p_{-}, J_{0 L}-J_{0 R}=h$ where $h$ is the (integer) helicity in the transverse plane. The generic physical state is generated from the basic level zero states

$$
\left|p_{+}, p_{-}, h ; n_{L}, n_{R}\right\rangle \otimes\left|p_{T}\right\rangle
$$

by the action of the negative modes of the $H_{4}^{L} \times H_{4}^{R} \times U(1)_{L}^{6} \times U(1)_{R}^{6}$ current algebra $\left(p_{T}\right.$ labels momentum on $\left.\mathbb{R}^{6}\right)$. Thus, taking into account the spectral flow, the physical state conditions in the bosonic string case are

$$
\begin{aligned}
& L_{0}=p_{+}^{L} p_{-}^{L}+p_{+}^{L} n_{L}+\frac{1}{2} p_{+}^{L}\left(1-p_{+}^{L}\right)+\frac{1}{2} p_{\perp}^{2}+N_{L}=1 \\
& \bar{L}_{0}=p_{+}^{R} p_{-}^{R}+p_{+}^{R} n_{R}+\frac{1}{2} p_{+}^{R}\left(1-p_{+}^{R}\right)+\frac{1}{2} p_{\perp}^{2}+N_{R}=1
\end{aligned}
$$

where $p_{+}^{L}=p_{+}+w_{L}, p_{+}^{R}=p_{+}+w_{R}, p_{-}^{L}=p_{-}-h / 2, p_{-}^{R}=p_{-}+h / 2$, and $n_{L, R}, N_{L, R}$ are defined as below (3.14).

\footnotetext{
${ }^{5}$ This is not exactly the light-cone gauge since here states with $p_{+}=0$ are retained.
} 
Let us first assume that neither of the light-cone directions are compact. The spectral flow has to be symmetric, hence $p_{+}^{L}=p_{+}^{R}=p_{+}$. The matching condition follows by taking the difference $L_{0}-\bar{L}_{0}$ :

$$
p_{+}\left(h-\operatorname{sgn}\left(p_{+}\right)\left(n_{L}-n_{R}\right)\right)=N_{L}-N_{R}
$$

Since in any consistent CFT the conformal spin must be integer, and since $p_{+}$is continuous, we must impose from the start the matching condition $h-\operatorname{sgn}\left(p_{+}\right)\left(n_{L}-\right.$ $\left.\left.n_{R}\right)\right)=0$, identical to the tree-level answer. This further implies $N_{L}=N_{R}$ for the left and right excitation numbers of the string. Taking now the sum $L_{0}+\bar{L}_{0}$, we find the physical mass spectrum of the closed bosonic string in the Nappi-Witten background,

$$
2 p_{+} p_{-}+\left|p_{+}\right|\left(n_{L}+n_{R}\right)+\left|p_{+}\right|\left(1-\left|p_{+}\right|\right)+p_{\perp}^{2}+N_{L}+N_{R}-2=0,
$$

In this expression, $p_{+}$runs from $-\infty$ to $+\infty$, keeping in mind the fact that states outside the range $-1 \leq p_{+} \leq 1$ are "long string" states obtained by spectral flow from the domain $\left|p_{+}\right|<1$. This differs by a quadratic term in $p_{+}$from the treelevel dispersion relation (2.13). Reinstating units, the correction is $p_{+}\left(1-\mu \alpha^{\prime} p_{+}\right)$, hence it can be thought as a one-loop correction in the $\sigma$ model. Its effect is to tilt the spectrum in the $\left(p_{+}, p_{-}\right)$plane. The quadratic correction cancels in the supersymmetric case (e.g. relevant for the NS5-brane) due to the fact that the fermions $\psi$ and $\bar{\psi}$ are twisted by the same amount as their bosonic partner $w$ so that the supercurrent remains invariant [19]. This structure should be contrasted with the case of Ramond backgrounds, where the energy of a level $n$ oscillator includes an infinite number of $\alpha^{\prime}$ corrections upon expanding the square root $\sqrt{n^{2}+\left(\mu \alpha^{\prime} p_{+}\right)^{2}}$ : this can be traced to the fact that perturbative strings are neutral under the Ramond background, whereas here the NS background gives an additional contribution to the mass.

If we now allow for a compact $u$ direction, the momentum $p_{-}$becomes quantized in integer units (since the radius of $u$ is fixed to be 1 ), and the spectral flows $w_{L}$ and $w_{R}$ can be different integers. Let us denote $w=\left(w_{L}+w_{R}\right) / 2$. The lhs of the matching relation (3.27) receives an extra contribution

$$
\left(w_{L}-w_{R}\right)\left(p_{-}-p_{+}-w+\frac{1}{2}\left(1+n_{L}+n_{R}\right)\right)
$$

allowing for more possibilities for level matching. If on the other hand the coordinate $v$ is made compact, the momentum $p_{+}$becomes quantized. In order to keep the conformal spin integer, the radius of $v$ should be fixed to $2 \pi$. It is then possible to satisfy the matching relation (3.27) with $N_{L} \neq N_{R}$. Finally, let us comment on type I states, with $p_{+}=0$. Except for the tachyon, these states can carry only momentum along $v$, that is along the wave itself. In fact, they can be thought of as the constituents of the wave, in agreement with the fact that $p_{+}=0$ encode the vacuum structure in light cone quantization. 


\subsection{The fate of discrete symmetries}

The pp-wave backgrounds $H_{D}$ threaded by NS flux

$$
d s^{2}=2 d u d v+\sum_{i=1}^{\frac{D}{2}} d w^{i} d \bar{w}^{i}-\frac{1}{4} \mu^{2}\left(\sum_{i=1}^{D / 2} w^{i} \bar{w}^{i}\right) d u^{2} \quad, \quad H=\mu d u \wedge \sum_{i=1}^{D / 2} d w^{i} \wedge d \bar{w}^{i}
$$

have a geometrical discrete (permutation) symmetry $S_{D / 2}$ that permutes the $D / 2$ 2-planes. This symmetry is acting on the $H_{D}$ symmetry algebra as an external automorphism, permuting pairs of raising and lowering operators

$$
P_{i}^{ \pm} \leftrightarrow P_{j}^{ \pm}
$$

The ground-state of a generic type II/III representation (corresponding to the harmonic oscillator ground state) can be written as

$$
R_{I I, I I I}^{\text {ground-state }}=\left|\prod_{i=1}^{D / 2} H_{p_{+}}^{i}\right\rangle
$$

where $H_{p_{+}}^{i}$ is the twist field of the i-th plane. Therefore the $S_{D / 2}$ symmetry is unbroken in the quantum theory. In the special case $D=4$ corresponding to the Penrose limit of $A d S_{3} \times S^{3}$ (near horizon region of NS5-F1 intersection) the symmetry is $Z_{2}$ and it acts in a similar fashion as the $Z_{2}$ that interchanges $A d S_{5} \leftrightarrow S^{5}$.

\section{Null holography}

Having the dynamics of particles and strings under control, we now turn to the main motivation of this paper, namely to investigate whether a holographic description analogous to AdS/CFT can hold in these homogeneous wave backgrounds. A necessary condition is to set up a well defined Cauchy problem, in order to relate boundary data on a holographic screen to bulk fields. This is clearly not sufficient, e.g. in AdS one may have chosen time as a candidate holographic direction, however one would have had to introduce two boundaries at $t= \pm \infty$, as in the dS case.

In the pp-wave background (11.1), the light-cone coordinate $u$ seems to be the only Killing vector leading to a well-defined Cauchy problem. For example, the oscillatory motion along $v$ precludes to use it as a time coordinate, since fields at a given $v_{0}$ will propagate both forward and backward. Another way to see that the propagation along $v$ is problematic is that the kinetic term $\partial_{v}^{2}$ in the wave equation (2.10) is singular at $x_{1}^{2}+x_{2}^{2}=0$, so that only wave packets with no support at the bottom of the potential may be propagated. Alternatively, one may be tempted to think of the radial direction in transverse plane as the holographic direction, since, in analogy with AdS, massive particles are confined along this direction. However, this is also true 
of the massless ones (with the exception of the $p_{+}=0$ modes, propagating strictly along the wave), so that this direction is effectively compact, and not a boundary. On the other hand, the $u$ direction is non-compact with smooth propagation and one can construct well-defined in and out-states.

Another crucial ingredient in the AdS/CFT correspondence is the relation between holographic flow in the bulk and RG flow in the dual gauge theory (this may not be a generic feature of holography, however we will conservatively assume that this is true). This relation is particularly obvious in the Poincaré patch, where the metric is the analytic continuation of the one on the Poincaré upper half plane,

$$
d s^{2}=\left(-d t^{2}+d x_{i}^{2}+d z^{2}\right) / z^{2}
$$

Going to the boundary $z \rightarrow 0$ makes an infinite rescaling of the metric, hence corresponds to the UV of the gauge theory, while the IR flow corresponds to $z \rightarrow \infty$.

\subsection{Holography in the Poincaré patch}

Here we show that the pp-wave background can be put in a form very similar to the Poincaré patch of AdS (4.1), where the light-cone coordinate $u$ now generates the RG flow. Notice first that like all the other maximally symmetric Lorentzian spaces, the pp-wave background (1.1) is conformally flat. The conformal factor making the curvature vanish is easily found to be $1 / \sin ^{2}\left(\left(u-u_{0}\right) / 2\right)$ for arbitrary $u_{0}$. Each interval of length $2 \pi$ can therefore be mapped through a non-singular conformal transformation to Minkowski space. The change of coordinates making the space manifestly conformal to Minkowski space is easily found: starting from the coordinates in (1.1), define

$$
x^{+}=-\cot (u / 2), \quad v=x^{-}-\frac{1}{2}\left(y_{1}^{2}+y_{2}^{2}\right) \sin u, \quad x_{i}=2 y_{i} \sin (u / 2)
$$

The metric and flux then read

$$
d s^{2}=\frac{4}{1+x^{+2}}\left(d x^{+} d x^{-}+d y_{1}^{2}+d y_{2}^{2}\right), \quad H=\frac{1}{\left(1+x^{+2}\right)^{2}} d x^{+} d y_{1} d y_{2}
$$

A motion along the $x^{+}$direction therefore appears to rescale the metric of a fixed $x^{+}$slice. Our claim is that there should exist a holographic dual to string theory in a pp-wave background of type $H_{D}$, with degrees of freedom living on the degenerate space described by the $\left(x^{-}, y_{i}\right)$ coordinates, together with the other flat transverse coordinates. The correlators in the holographic dual are S-matrix elements relating the incoming states at $x^{+}=-\infty$ to the outgoing states at $x^{+}=+\infty$. The holographic direction is then the null direction $x^{+}$, to be contrasted with the space-like (resp. time-like) direction in AdS (resp. dS).

This claim requires some qualifications and comments. Firstly, in contrast to AdS, the conformal factor $1 /\left(1+x^{+^{2}}\right)$ never blows up. It reaches a maximum at 
$x^{+}=0$, which implies that the UV is effectively cut-off in the holographic dual. We view this as an indication that the dual holographic degrees of freedom are nonlocal - possibly a string theory of a new type. On the other hand, as $x^{+} \rightarrow \pm \infty$, the conformal factor goes to zero, corresponding to the horizon of the patch, so these regions should correspond to the IR of the gauge theory. Attempts to define holography on the horizon are not unprecedented [39], and this is also the approach we follow here.

Next, propagation along a null coordinate $x^{+}$involves a first-order differential operator in $x^{+}$. This forbids to impose boundary conditions at the two boundaries $x^{+}= \pm \infty$ at the same time, unlike the de Sitter case. The dichotomy in AdS between non-normalizable solutions corresponding to operator insertions and normalizable ones describing the vacuum [40] seems also to be lost. Only the first kind is kept, since the vacuum structure corresponds to $p_{+}=0$ states which cannot propagate along $x^{+}$. In fact, the Cauchy problem with a single slice at fixed $x^{+}$is ill-defined: as in Minkowski space, it is necessary to include boundary conditions on the other side of the wedge, at fixed $x^{-}$. The vacuum structure should therefore be specified on an extra "holographic screen" at fixed $x^{-}$.

Finally, the conformal coordinate system only covers a patch $0<x^{+}<2 \pi$ of the global geometry (1.1) (or any translation thereof), which we may call the Poincaré patch of the pp-wave geometry. Even though we have seen that points at $u=0$ and $u=2 \pi$ were an infinite proper distance away unless $x_{i}= \pm x_{i}^{\prime}$, this truncation may be inconsistent. One would there have to go back to the global coordinates $\left(u, v, x_{i}\right)$, where we still expect the coordinate $u$ to play the rôle of the holographic direction.

\subsection{Geometry of conformal coordinates}

In order to evaluate the validity of this claim, let us first discuss some aspects of the pp-wave geometry in these coordinates. To start with, the Killing vectors corresponding to the action of $H_{4 L} \times H_{4 R}$ read

$$
\begin{aligned}
K & =\partial_{-} \\
J_{L} & =\frac{1+x^{+2}}{2} \partial_{+}-\frac{y_{1}^{2}+y_{2}^{2}}{2} \partial_{-}+\frac{1}{2}\left(x^{+} y_{1}-y_{2}\right) \partial_{1}+\frac{1}{2}\left(x^{+} y_{2}+y_{1}\right) \partial_{2} \\
J_{R} & =\frac{1+x^{+2}}{2} \partial_{+}-\frac{y_{1}^{2}+y_{2}^{2}}{2} \partial_{-}+\frac{1}{2}\left(x^{+} y_{1}+y_{2}\right) \partial_{1}+\frac{1}{2}\left(x^{+} y_{2}-y_{1}\right) \partial_{2} \\
P_{i L} & =y_{i} \partial_{-}-\frac{x^{+}}{2} \partial_{i}-\frac{1}{2} \epsilon_{i j} \partial_{j} \\
P_{i R} & =y_{i} \partial_{-}-\frac{x^{+}}{2} \partial_{i}+\frac{1}{2} \epsilon_{i j} \partial_{j}
\end{aligned}
$$

where $\partial_{i}:=\partial / \partial y_{i}$ and $\partial_{ \pm}:=\partial / \partial x^{ \pm}$. The translations along the three flat directions $\left(v, y_{1}, y_{2}\right)$ correspond to the generators $K, P_{2 L}-P_{2 R}$ and $P_{1 R}-P_{1 L}$ respectively. The 
Laplacian takes the form

$$
\Delta=\frac{1}{4}\left(1+x^{+2}\right)\left(4 \partial_{+} \partial_{-}+\partial_{1}^{2}+\partial_{2}^{2}\right)-x^{+} \partial_{-}
$$

where now $\partial_{i}=\partial / \partial y_{i}$. In particular, we see that the motion is not confined any more in the transverse directions $y_{1}, y_{2}$. The geodesics (2.8) are in fact mapped to lines of arbitrary constant transverse position,

$$
x^{+}(\tau)=-2 \cot \left(p_{+} \tau\right), \quad x^{-}=p^{2} \tau / p_{+}, \quad y_{i}=p_{i} / p_{+}
$$

Remarkably, the slices of constant $x^{+}$of the metric (4.3) are invariant under all Killing vectors except for

$$
2 P_{-}=J_{L}+J_{R}=\left(1+x^{+^{2}}\right) \partial_{+}-\left(y_{1}^{2}+y_{2}^{2}\right) \partial_{-}+x^{+}\left(y_{1} \partial_{1}+y_{2} \partial_{2}\right)
$$

This is the generator we want to identify with the holographic direction: in terms of the original coordinates (1.1), it is simply the translation along the null $u$ direction. Assuming that the holographic dual indeed lives along a slice of fixed $x^{+}$, we will have to find a realization of the generator $P_{-}$that does not involve the normal direction $x^{+}$: we will come back to this point shortly.

\subsection{Generalized conformal wave background}

Having obtained the simple form (4.3) for the metric of the homogeneous pp-wave in conformal coordinates, it is very natural to consider the more general $D$-dimensional conformally flat (new) background with a null Killing vector,

$$
d s^{2}=\frac{1}{f^{2}\left(x^{+}\right)}\left(d x^{+} d x^{-}+\sum d y_{i}^{2}\right), \quad H=\sqrt{f^{\prime \prime} / f^{5}} d x^{+} \wedge \omega
$$

This solves the string equations of motion, as long as the function $f$ is convex, $f^{\prime \prime}>0$ (as usual, $\omega$ is a constant symplectic form on the $(D-2)$ dimensional transverse space). The choice $f=\sqrt{1+x^{+2}} / 2$ corresponds to the homogeneous wave background (4.3). The case where $f$ is constant is of course flat Minkowski space. The case $f=x^{+}$corresponds to the large $x^{+}$limit of the homogeneous wave background, and is also the same as flat space up to change of coordinates $\left(x^{+}, x^{-}, y^{i}\right) \rightarrow\left(-1 / x^{+}, x^{-}+y_{i}^{2} / x^{+}, y^{i} / x^{+}\right)$. The background (4.3) is therefore asymptotically flat at $x^{+}= \pm \infty$, and one may be able to define an S-matrix relating the in-states at $x^{+}=-\infty$ to the out-states at $x^{+}=+\infty$, after they interact with the wave around $\left|x^{+}\right| \sim 1^{6}$. A further generalization of the class (4.8) would be to consider the conformal rescaling of a Lorentzian space with a null Killing vector $\partial_{+}$, by a conformal factor depending on the null coordinate $x^{+}$.

\footnotetext{
${ }^{6}$ Note that one may also consider convex $f$-profiles that interpolate between constant $f$ at $-\infty$ and linear at $+\infty$, or reverse.
} 
The geodesics in the generalized background (4.8) are easily computed, and read

$$
\begin{aligned}
y_{i}(\tau) & =y_{i}+\frac{p_{i}}{p_{+}}\left(x^{+}(\tau)-x^{+}\right) \\
x^{-}(\tau) & =x^{-}-\frac{p_{i}^{2}}{p_{+}^{2}}\left(x^{+}(\tau)-x^{+}\right)+\frac{p_{-}}{p_{+}} \int_{x^{+}{ }_{0}}^{x^{+}(\tau)} \frac{d u}{f^{2}(u)}
\end{aligned}
$$

where the proper time is related to the light-cone time by $p_{+} \tau=\int_{x^{+}}^{x^{+}} d u / f^{2}(u)$. The motion in the transverse directions $y^{i}$ is therefore free as expected. It is interesting to note that when the integral $\int d u / f^{2}$ converges, as in the pp-wave case of interest, the motion is in fact massless at late times, irrespective of the mass of the particle in the bulk. Another interesting case is when $f\left(x^{+}\right) \rightarrow 1$ at $\pm \infty$ : the coordinate $v$ then experiences a translation by $\left(p_{-} / p_{+}\right) \int_{-\infty}^{\infty}\left(1 / f^{2}-1\right) d u$, as in standard shock wave backgrounds.

\subsection{Null Cauchy problem}

If indeed $x^{+}$is the holographic direction, one should be able to recover the bulk configuration from "initial" data at the boundary $x^{+}=x_{0}^{+}$(eventually taking the limit $\left.x_{0}^{+} \rightarrow-\infty\right)$. We will now work out the eigenmodes and propagator in the background (4.8). More information and derivations are presented in appendix A. Eigenmodes of the Laplacian

$$
\Delta=f^{2}\left(\Delta_{T}+4 \partial_{+} \partial_{-}\right)-(D-2)\left[f^{2}\right]^{\prime} \partial_{-}
$$

(where $\Delta_{T}=\partial_{i} \partial_{i}$ is the Laplacian on the $(D-2)$ transverse coordinates) are easily computed for arbitrary $f$ profile,

$$
\phi=f^{\frac{D-2}{2}}\left(x^{+}\right) \exp \left(i\left(p_{+} x^{-}+p_{i} y_{i}-\frac{p_{i}^{2}}{4 p_{+}} x^{+}\right)-i \frac{m^{2}}{4 p_{+}} \int_{-\infty}^{x^{+}} \frac{d u}{f^{2}(u)}\right)
$$

up to a normalization factor. Notice that the phase is simply the one for a massless wave in Minkowski space-time with momentum $p_{-}=-p_{i}^{2} / 4 p_{+}$, up to an additive phase shift proportional to the integral of the conformal factor. The total phase shift after crossing the curved background at $\left|x^{+}\right| \sim 1$ is

$$
\Delta \varphi=\frac{m^{2}}{4 p_{+} \mu} \int_{-\infty}^{\infty} \frac{d u}{f^{2}(u)}=\frac{\pi m^{2}}{\mu p_{+}}
$$

which we evaluated in the homogeneous pp-wave of interest, after reinstating the mass scale $\mu$. This should be compared with the phase shift in Minkowski space

$$
\frac{m^{2}}{4 p_{+} \mu} \int_{-\infty}^{\infty} d u=\frac{m^{2} \Delta x^{+}}{4 \mu p_{+}}
$$


which diverges linearly. The boundary to bulk propagator can be found by adjusting the normalization so that $\phi\left(u, p_{+}, p_{i}\right) \rightarrow 1$ as $u \rightarrow u_{0}$. Going back to position variables in transverse space, we get

$$
\begin{aligned}
G\left(x^{+}, x^{-}, y_{i} ; x_{0}^{+}\right)= & \int_{-\infty}^{\infty} \frac{d p_{-}}{(2 \pi)^{D-1}}\left(\frac{4 p_{+} f\left(x^{+}\right)}{\left(x^{+}-x_{0}^{+}\right) f\left(x_{0}^{+}\right)}\right)^{\frac{D-2}{2}} \\
& \exp \left(i p_{+} \frac{x^{-}\left(x^{+}-x_{0}^{+}\right)+y_{i}^{2}}{x^{+}-x_{0}^{+}}-i \frac{m^{2}}{4 p_{+}} \int_{x_{0}^{+}}^{x^{+}} \frac{d u}{f^{2}(u)}\right)
\end{aligned}
$$

The integral over $p_{+}$is of the Bessel type, and yields

$G\left(x^{+}, x^{-}, y_{i} ; x_{0}^{+}\right)=\theta\left(-r^{2}\right) \frac{\left(x^{+}-x_{0}^{+}\right)^{\frac{(2-D)}{2}}}{(2 \pi)^{D / 2} i^{D-1}}\left(\frac{f\left(x^{+}\right)}{f\left(x_{0}^{+}\right)}\right)^{\frac{D-2}{2}}\left(\frac{\gamma m^{2}}{r^{2}}\right)^{\frac{D}{4}} J_{D / 2}\left(\sqrt{\frac{\gamma m^{2} r^{2}}{\left(x^{+}-x_{0}^{+}\right)}}\right)$

where $\theta(x)$ is the Heaviside function, $r^{2}$ is the invariant distance from $\left(x^{+}, x^{-}, y_{i}\right)$ to $\left(x_{0}^{+}, 0,0\right)$ in Minkowski space, $r^{2}=\left(x^{+}-x_{0}^{+}\right) x^{-}+y_{1}^{2}+y_{2}^{2}$, and $\gamma$ summarizes the effect of the curved background,

$$
\gamma=\frac{1}{x^{+}-x_{0}^{+}} \int_{x_{0}^{+}}^{x^{+}} \frac{d u}{f^{2}(u)}>0
$$

In particular for $f=1$ we recover the standard boundary to bulk propagator of Minkowski space, $\gamma=x^{+}-x_{0}^{+}$. The main effect of the wave background is therefore to stretch the invariant distance $r^{2} \rightarrow \gamma r^{2}$, i.e. to introduce a refractive index $\gamma$. We also present the bulk propagator which will be useful in holographic computations

$$
\begin{gathered}
G\left(x^{+}, x^{-}, y ; x^{+^{\prime}}, x^{-^{\prime}}, y^{\prime}\right)=2^{(D-6) / 2}\left[f\left(x^{+}\right) f\left(x^{+^{\prime}}\right)\right]^{\frac{D-2}{2}} H\left(x^{+}-x^{+^{\prime}}\right) \\
\times\left(\frac{\pi^{2} m^{2} \gamma}{\left(x^{+}-x_{0}^{+}\right) x^{2}}\right)^{\frac{D-2}{4}} J_{\frac{D-2}{2}}\left(\sqrt{\frac{\gamma m^{2} r^{2}}{\left(x^{+}-x_{0}^{+}\right)}}\right)
\end{gathered}
$$

Our derivation of the boundary to bulk propagator so far has been casual and assumed special boundary conditions at $x^{-} \rightarrow \infty$. If order to understand better the content of holographic correspondence in pp-wave backgrounds, we need to allow for arbitrary states at $v=-\infty$. The careful analysis of [31] in the context of two-dimensional QED on the light cone can be transposed to our case as follows. Let us consider a solution $\phi$ of $\Delta-m^{2}=0$, specified by its values on the wedge $\left(x^{+}=x_{0}^{+}, x^{-}>x_{0}^{-}\right)$and $\left(x^{-}=x_{0}^{-}, x^{+}>x_{0}^{+}\right)$. Defining the normalized field $\phi_{-}=\phi f^{(2-D) / 2}$ and its canonical conjugate $\phi_{+}=\partial_{-} \phi_{-}$, the wave equation can be rewritten in the first order form

$$
\partial_{-} \phi_{-}=\phi_{+}, \quad 4 \partial_{+} \phi_{+}+\Delta_{T} \phi_{-}=\frac{m^{2}}{f^{2}} \phi_{-}
$$


This can be integrated to (see appendix B)

$$
\begin{gathered}
\phi_{+}\left(x^{+}, x^{-}, y\right)=\int_{-\infty}^{+\infty} \frac{d p_{+}}{2 \pi}\left[\int_{x_{0}^{-}}^{+\infty} d v e^{i p_{+}\left(v-x^{-}\right)} \exp \left(\frac{i}{4 p_{+}} \int_{x_{0}^{+}}^{x^{+}}\left(\frac{m^{2}}{f^{2}(u)}-\Delta_{T}\right) d u\right) \phi_{+}\left(x_{0}^{+}, v, y\right)\right. \\
\left.+\frac{i e^{-i p_{+}\left(x^{-}-x_{0}^{-}\right)}}{4 p_{+}} \int_{x_{0}^{+}}^{x^{+}} d u\left[\exp \left(\frac{i}{4 p_{+}} \int_{u}^{x^{+}}\left(\frac{m^{2}}{f^{2}\left(u^{\prime}\right)}-\Delta_{T}\right) d u^{\prime}\right)\right]\left(\frac{m^{2}}{f^{2}(u)}-\Delta_{T}\right) \phi_{-}\left(u, x_{0}^{-}, y\right)\right]
\end{gathered}
$$

where the integration prescription $p_{+} \rightarrow p_{+}+i \epsilon$ is understood. The conjugate field can be obtained as

$$
\begin{aligned}
& \phi_{-}\left(x^{+}, x^{-}, y\right)=\phi_{-}\left(x^{+}, x_{0}^{-}, y\right)+ \\
& +\int_{-\infty}^{+\infty} \frac{d p_{+}}{2 \pi}\left[\int_{x_{0}^{-}}^{+\infty} d v \frac{i e^{i p_{+}\left(v-x^{-}\right)}}{p_{+}} \exp \left(\frac{i}{4 p_{+}} \int_{x_{0}^{+}}^{x^{+}}\left(\frac{m^{2}}{f^{2}(u)}-\Delta_{T}\right) d u\right) \phi_{+}\left(x_{0}^{+}, v, y\right)\right. \\
& \left.-\frac{e^{-i p_{+}\left(x^{-}-x_{0}^{-}\right)}}{4 p_{+}^{2}} \int_{x_{0}^{+}}^{x^{+}} d u\left[\exp \left(\frac{i}{4 p_{+}} \int_{u}^{x^{+}}\left(\frac{m^{2}}{f^{2}\left(u^{\prime}\right)}-\Delta_{T}\right) d u^{\prime}\right)\right]\left(\frac{m^{2}}{f^{2}(u)}-\Delta_{T}\right) \phi_{-}\left(u, x_{0}^{-}, y\right)\right]
\end{aligned}
$$

It can be verified that the solution above asymptotes properly at the limits $x^{+} \rightarrow x_{0}^{+}$ and $x^{-} \rightarrow x_{0}^{-}$. The limit $x^{+} \rightarrow x_{0}^{+}$is fairly obvious from the formula above. The other limit is subtle and the $i \epsilon$ prescription is crucial. Hence the values of $\phi_{+}$at $x^{+}=x_{0}^{+}$and of $\phi_{-}$at $x^{-}=x_{0}^{-}$uniquely specify the field for all later $x^{+}$and $x^{-} .{ }^{7}$

Finally, let us consider the effect of taking the limit $x^{-}{ }_{0} \rightarrow-\infty$ on the result (4.19). Using (B.14) we get

$$
\begin{aligned}
& \phi_{+}\left(x^{+}, p_{+}, y\right)=\exp \left(\frac{i}{4 p_{+}} \int_{x_{0}^{+}}^{x^{+}}\left(\frac{m^{2}}{f^{2}(u)}-\Delta_{T}\right) d u\right) \phi_{+}\left(x_{0}^{+}, p_{+}, y\right)+2 \pi \delta\left(p_{+}\right) \times \\
& \times \int_{x_{0}^{+}}^{x^{+}} d u\left[\exp \left(\frac{i}{4 p_{+}} \int_{u}^{x^{+}}\left(\frac{m^{2}}{f^{2}\left(u^{\prime}\right)}-\Delta_{T}\right) d u^{\prime}\right)\right]\left(\frac{m^{2}}{f^{2}(u)}-\Delta_{T}\right) \phi_{-}(u,-\infty, y)
\end{aligned}
$$

This shows that non-zero $\phi\left(x^{+}, x^{-} \rightarrow-\infty, y\right)$ generates delta function singularities at $p_{+}=0$. Our discussion of holography will be restricted for simplicity to the case where $\phi$ vanishes at $x^{-} \rightarrow-\infty$. It would be interesting to develop techniques to regulate these contributions.

\subsection{Boundary symmetries and Ward identities}

At this stage, the boundary to bulk propagator provides us with a very concrete way to set up the holographic correspondence: in analogy with the $A d S_{3}$ case, we may

\footnotetext{
${ }^{7}$ In Appendix B we show that these expressions imply that there is no particle production in this background when $p_{+} \neq 0$, as expected on general grounds in backgrounds with a null Killing vector. This is in contrast to the constant electric field case considered in [31.
} 
think of the propagator $G\left(x^{+}, x^{-}, y_{i} ; x_{0}^{+}, x_{0}^{-}, y_{i 0}\right):=G\left(x^{+}, x^{-}-x_{0}^{-}, y_{i}-y_{i 0} ; x_{0}^{+}\right)$as the bulk closed string vertex associated to a holographic operator localized at $\left(x_{0}^{-}, y_{i 0}\right)$ on the boundary. The action of $H_{4 L} \times H_{4 R}$ on the bulk coordinate $\left(x^{+}, x^{-}, y_{i}\right)$ translates into an action on the holographic coordinate $\left(x_{0}^{-}, y_{i 0}\right)$.

As we have seen earlier, the solution to the Laplace equation in terms of the boundary value at $x^{+}=x_{0}^{+}$and zero on the $x_{0}^{-}=-\infty$ screen is

$$
\begin{aligned}
& \phi_{0}\left(x^{+}, x^{-}, y\right)=\int d p_{+} d p\left(\frac{f\left(x^{+}\right)}{f\left(x_{0}^{+}\right)}\right)^{\frac{D-2}{2}} \\
& \quad \times \exp \left[i\left(p_{+} x^{-}+p \cdot y\right)-i \frac{p^{2}}{4 p_{+}}\left(x^{+}-x_{0}^{+}\right)-i \frac{m^{2}}{4 p_{+}} \int_{x_{0}^{+}}^{x^{+}} \frac{d u}{f^{2}(u)}\right] \phi\left(p_{+}, p, x_{0}^{+}\right)
\end{aligned}
$$

We focus on $D=4$ where $f=\sqrt{1+x^{+2}} / 2$. The Killing vectors $\mathcal{L}_{i}$ corresponding to the action of $H_{4 L} \times H_{4 R}$ in the bulk were given in (4.5). Since they commute with the Laplacian, they can be commuted through the boundary to bulk propagator and act on the boundary field $\phi\left(p_{+}, p, x_{0}^{+}\right)$. We define the boundary action of the symmetry (in momentum space) as

$$
\begin{aligned}
& \mathcal{L}_{i} \phi_{0}\left(x^{+}, x^{-}, y\right)=\int d p_{+} d p\left(\frac{f\left(x^{+}\right)}{f\left(x_{0}^{+}\right)}\right)^{\frac{D-2}{2}} \\
& \quad \times \exp \left[i\left(p_{+} x^{-}+p \cdot y\right)-i \frac{p^{2}}{4 p_{+}}\left(x^{+}-x_{0}^{+}\right)-i \frac{m^{2}}{4 p_{+}} \int_{x_{0}^{+}}^{x^{+}} \frac{d u}{f^{2}(u)}\right] \hat{\mathcal{L}}_{i} \phi\left(p_{+}, p, x_{0}^{+}\right)
\end{aligned}
$$

From (4.5,4.22) we obtain

$$
\begin{aligned}
& \hat{K}=i p_{+} \\
& \hat{J}_{L}=i \frac{p_{+}}{2} \Delta_{p}-\frac{x_{0}^{+}}{2}\left(p_{i} \partial_{p_{i}}\right)+\frac{1}{2}\left(p_{1} \partial_{p_{2}}-p_{2} \partial_{p_{1}}\right)-i \frac{p^{2}}{8 p_{+}}\left(1+x_{0}^{+2}\right)-i \frac{m^{2}}{2 p_{+}}-\frac{x_{0}^{+}}{2} \\
& \hat{J}_{R}=i \frac{p_{+}}{2} \Delta_{p}-\frac{x_{0}^{+}}{2}\left(p_{i} \partial_{p_{i}}\right)-\frac{1}{2}\left(p_{1} \partial_{p_{2}}-p_{2} \partial_{p_{1}}\right)-i \frac{p^{2}}{8 p_{+}}\left(1+x_{0}^{+2}\right)-i \frac{m^{2}}{2 p_{+}}-\frac{x_{0}^{+}}{2} \\
& \hat{P}_{i L}=-p_{+} \partial_{p_{i}}-i \frac{x_{0}^{+}}{2} p_{i}-\frac{i}{2} \epsilon^{i j} p_{j}, \quad \hat{P}_{i R}=-p_{+} \partial_{p_{i}}-i \frac{x_{0}^{+}}{2} p_{i}+\frac{i}{2} \epsilon^{i j} p_{j}
\end{aligned}
$$

where $\Delta_{p}=\partial_{p_{i}} \partial_{p_{i}}$. It can be checked that $\mathcal{L}_{i}$ satisfy the conjugate algebra of $\hat{\mathcal{L}}_{i}$. As expected, the generators are singular when $p_{+}=0$. Interestingly, the dependence on the location $x_{0}^{+}$of the boundary can be disposed of by performing the non-local field redefinition

$$
\phi\left(p_{+}, p_{1}, p_{2}\right)=\tilde{\phi}\left(p_{+}, p_{1}, p_{2}\right) \exp \left(-i \frac{p_{1}^{2}+p_{2}^{2}}{4 p_{+}} x_{0}^{+}\right)
$$


The generators then take the simpler form

$$
\begin{aligned}
\tilde{K} & =i p_{+} \\
\tilde{J}_{L} & =i \frac{p_{+}}{2} \Delta_{p}-\frac{i}{8 p_{+}}\left(p_{1}^{2}+p_{2}^{2}+4 m^{2}\right)+\frac{1}{2}\left(p_{1} \partial_{p_{2}}-p_{2} \partial_{p_{1}}\right) \\
\tilde{J}_{R} & =i \frac{p_{+}}{2} \Delta_{p}-\frac{i}{8 p_{+}}\left(p_{1}^{2}+p_{2}^{2}+4 m^{2}\right)-\frac{1}{2}\left(p_{1} \partial_{p_{2}}-p_{2} \partial_{p_{1}}\right) \\
\tilde{P}_{i L} & =-p_{+} \partial_{p_{i}}-\frac{i}{2} \epsilon^{i j} p_{j}, \quad \tilde{P}_{i R}=-p_{+} \partial_{p_{i}}+\frac{i}{2} \epsilon^{i j} p_{j}
\end{aligned}
$$

Transforming back to position space using Fourier transform

$$
\phi\left(x_{0}^{+}, x^{-}, y\right)=\int d p_{+} d p \exp \left[i\left(p_{+} x^{-}+p \cdot y\right)\right] \tilde{\phi}\left(p_{+}, p, x_{0}^{+}\right)
$$

we obtain the boundary representation of the $H_{D} \times H_{D}$ symmetry,

$$
\begin{aligned}
\tilde{K} & =\partial_{-} \\
\tilde{J}_{L}+\tilde{J}_{R} & =-y_{i}^{2} \partial_{-}+\frac{1}{4}\left(4 m^{2}-\Delta\right) \partial_{-}^{-1} \\
\tilde{J}_{L}-\tilde{J}_{R} & =y_{1} \partial_{2}-y_{2} \partial_{1} \\
\tilde{P}_{i L} & =y_{i} \partial_{-}-\frac{1}{2} \epsilon_{i j} \partial_{j}, \quad \tilde{P}_{i R}=y_{i} \partial_{-}+\frac{1}{2} \epsilon_{i j} \partial_{j}
\end{aligned}
$$

where $\partial_{-}^{-1}=\int d x^{-}, \partial_{-}^{-1} \partial_{-}=1$. This result can be generalized to arbitrary even $D$ by replacing $J_{L}+J_{R}$ by the rotation in all 2-planes simultaneously.

The formulae (4.27) thus describe the action of the bulk isometries on the boundary data. They correspond to symmetries that must be satisfied by the correlation functions of the putative dual gauge theory. They suggest the existence of a novel class of (possibly non-local) field theories where conformal invariance is replaced by invariance under the (super) Heisenberg-type group $H_{D}^{L} \times H_{D}^{R}$. The analogue of the conformal dimension is the mass $m^{2}$, which is also the Casimir of this representation. The holographic generator is the combination $J_{L}+J_{R}$ which is nothing but the Hamiltonian of the harmonic oscillator with frequency $\left|\partial_{-}\right| / 2$. Note in particular that the rescaling operator appearing in the last term of the bulk generator (4.7) has disappeared in the process of going to the boundary, due to the non-local field redefinition (4.24). In terms of the new field $\tilde{\phi}$, the holographic generator does not appear to involve a RG flow any more. Alternatively, one may have located the holographic screen at $x_{0}^{+}=0$, i.e. as close to the UV regime as one can gets, so that the field redefinition (4.24) would not be necessary. Since the conformal factor attains a maximum at $x^{+}=0$, one recovers the conclusion that the holographic generator should not involve any rescaling of the transverse coordinates.

It is interesting to determine the consequences of the Ward identities associated to this symmetry on the $n$-point functions correlators. Invariance under the generators $K$ and $P_{i L}-P_{i R}$ enforces momentum conservation in all directions $x^{-}, y_{i}$. 
For the two-point function, invariance under $P_{i L}+P_{i R}$ then implies that the correlator is independent of the transverse momenta $p_{i}$. Invariance under $J_{L}, J_{R}$ requires the masses of the two fields to be the same. The most general two-point function consistent with the symmetry is therefore

$$
\left\langle\phi_{m_{1}}\left(p_{+}, p\right) \phi_{m_{2}}\left(q_{+}, q\right)\right\rangle=f_{2}\left(p_{+}\right) \delta\left(p_{+}+q_{+}\right) \delta^{(2)}(p+q) \delta_{m_{1}, m_{2}}
$$

where $f_{2}\left(p_{+}\right)$is an arbitrary function of $p_{+}$. Of course, this discussion cannot rule out contact contributions at $p_{+}=0$, where the symmetry is ill-defined. The constraints on the 3-point function are further analyzed in Appendix D.

\subsection{Two-point and three-point functions}

Let us now try and derive the boundary correlators following the same logic as in the AdS/CFT case. There, the prescription was that the classical action of the solution of the bulk equations of motion satisfying appropriate boundary conditions, gave the generating functional for the off-shell correlation functions of the boundary operators [41]. We will follow the same logic here, and present a sample computation for a selfinteracting massive scalar field in the bulk in order to illustrate our methodology. Of course, a full-fledged computation should involve the whole supergravity multiplet in the bulk, or rather the whole tower of string states, which we will leave for future work. As discussed below (4.21), we will also for simplicity assume zero boundary conditions on the holographic screen at $x^{-} \rightarrow-\infty$. These are the only boundary conditions that leave the $H_{4 L} \times H_{4 R}$ symmetry unbroken in the boundary theory. It would be interesting to extend the computation below to the more general case, in order to study the effect on the correlators of changing the vacuum.

Let us therefore consider a self-interacting minimally-coupled massive scalar field in the bulk, with action

$$
S=-\frac{1}{2} \int d^{D} x \sqrt{-g}\left(g^{\mu \nu} \partial_{\mu} \phi \partial_{\nu} \phi+m^{2} \phi^{2}+\frac{2 \lambda}{3} \phi^{3}\right)
$$

The equations of motion $\left(\Delta-m^{2}\right) \phi=\lambda \phi^{2}$ can be solved perturbatively in $\lambda$ by expanding $\phi=\phi_{0}+\lambda \phi_{1}+\mathcal{O}\left(\lambda^{2}\right)$ to obtain

$$
\left(\Delta-m^{2}\right) \phi_{0}=0 \quad, \quad\left(\Delta-m^{2}\right) \phi_{1}=\phi_{0}^{2}
$$

We now evaluate the action $S=S_{0}+\lambda S_{1}+\mathcal{O}\left(\lambda^{2}\right)$ on the classical solution in order

to obtain the boundary correlators. This amounts to computing S-matrix transition amplitudes between $x^{+}=-\infty$ and $x^{+}=+\infty$. As usual, the on-shell action reduces 
to boundary terms,

$$
\begin{aligned}
S_{0} & =-\frac{1}{2} \int d^{D} x \sqrt{-g}\left(g^{\mu \nu} \partial_{\mu} \phi_{0} \partial_{\nu} \phi_{0}+m^{2} \phi_{0}^{2}\right) \\
& =-\int d x^{+} d x^{-} d^{D-2} y\left[\partial_{+}\left(f^{2-D} \phi_{0} \partial_{-} \phi_{0}\right)+\partial_{-}\left(f^{2-D} \phi_{0} \partial_{+} \phi_{0}\right)\right] \\
S_{1} & =-\lambda \int d^{D} x \sqrt{-g}\left(g^{\mu \nu} \partial_{\mu} \phi_{0} \partial_{\nu} \phi_{1}+m^{2} \phi_{0} \phi_{1}+\frac{1}{3} \phi_{0}^{3}\right) \\
& =-\frac{2 \lambda}{3} \int\left[2 \partial_{+} \partial_{-}\left(f^{2-D} \phi_{0} \phi_{1}\right)+\partial_{+}\left(f^{2-D} \phi_{1} \partial_{-} \phi_{0}\right)+\partial_{-}\left(f^{2-D} \phi_{1} \partial_{+} \phi_{0}\right)\right]
\end{aligned}
$$

This is evaluated in detail in Appendix C. Remarkably, the first order correction $S_{0}$ vanishes, implying a vanishing two-point function for the boundary field $\phi$. As we explain in Appendix D, this is consistent with the Ward identities, although the latter would allow a more general 2-point function. The three-point amplitude is however non-trivial. It can be rendered finite by a wave function renormalization $\phi_{0} \rightarrow \phi_{0} f\left(x_{0}^{+}\right)^{\frac{2-d}{2}}$. After amputating the renormalized sources and momentum conserving $\delta$-functions, the three-point amplitude is given by

$$
\begin{gathered}
F_{3}(p, q, r)=\lambda \frac{(2 \pi)^{D-1}}{12} \int_{x_{0}^{+} \rightarrow-\infty}^{\infty} d u f(u)^{\frac{D-6}{2}} \exp \left[-\frac{i m^{2}}{4}\left(\frac{1}{p_{+}}+\frac{1}{q_{+}}+\frac{1}{r_{+}}\right) \tilde{\gamma}\left(u, x_{0}^{+}\right)\right] \\
\times \exp \left[-\frac{i}{4}\left(\frac{p^{2}}{p_{+}}+\frac{q^{2}}{q_{+}}+\frac{r^{2}}{r_{+}}\right)\left(u-x_{0}^{+}\right)\right]
\end{gathered}
$$

with

$$
\tilde{\gamma}\left(x^{+}, x_{0}^{+}\right) \equiv \int_{x_{0}^{+}}^{x^{+}} \frac{d u}{f^{2}(u)}
$$

It can be checked that this result is compatible with the Ward identity (D.17). Extending the domain of integration to the whole space-time (i.e. integrating from $u=-\infty$ to $u=+\infty$ in global coordinates), the same result is obtained, multiplied by an extra delta function enforcing conservation of $m^{2} / p_{+}$modulo integers. This is in agreement with the conservation of $p_{-}$in global coordinates. The computation of higher amplitudes is in principle straightforward. It would be interesting to understand the effect of other boundary conditions at $x^{-} \rightarrow-\infty$ on these correlators.

\section{Summary, conclusions and open problems}

In this paper we have studied aspects of string propagation and holography homogeneous pp-wave backgrounds. These are exact string embeddings of the maximally symmetric Cahen-Wallach spaces which, in the case where there are supported by Neveu-Schwarz flux, can also be thought of as the group manifold of the extended Heisenberg group $H_{D}$. They arise as Penrose limits of the near horizon region of the 
the NS5-brane (blown up around a geodesic spinning around $S^{3}$ ) (for $H_{4}$ ), $A d S_{3} \times S_{3}$ $\left(H_{6}\right)$ as well as higher intersections of two NS5-branes with fundamental strings $\left(H_{8}, H_{10}\right)$. In addition, they are interesting tractable exemples of time-dependent backgrounds in string theory.

In the first part of this paper, we have discussed aspects of string propagation in these backgrounds, elaborating on earlier studies [17, 19]. The study of the geodesics and solutions of the wave equation has shown that the natural time variable is the null coordinate $x^{+}$. An observer co-moving with the wave has $x^{+}=$constant. Time slices with $\Delta x^{+}=2 \pi \mathbb{Z}$ have been shown to be an infinite proper distance away from each other, except for coinciding positions in transverse space.

We have subsequently discussed the covariant quantization of the NSR string in these backgrounds, using in particular the free-field resolution of the current algebra which enables us to construct vertex operators for all the affine representations. Type I states correspond to modes with $p_{+}=0$, travelling with the wave. They would be invisible in light-cone quantization. Their vertex operators are simple exponentials. They are massless and interact among themselves as in flat space, leading to only type I states in intermediate channels.

Type II and III states are localized at the origin in the transverse $D-2$ dimensional space, and their vertex operators are constructed in terms of twist fields of free complex bosons by an angle $2 \pi p_{+}$, in general irrational. They have non-trivial interactions, and it is an open problem to calculate their S-matrix. Solving the associated Knizhnik-Zamolodchikov equations is the most direct approach to this problem.

Since twist fields only exist for $0<1<p_{+}$, the infinite range of $p_{+}$is generated by the spectral flow(s) of the $H_{D}$ current algebra. The new states are associated with long strings winding (and oscillating under the influence of the wave) in the transverse harmonic potential. At integer values of $p_{+}$, they are also long strings that expand linearly with light-cone time in the transverse directions. For specific value of the energy, they exist as static circular strings, and can change their radius at no cost of energy. The inclusion of spectral flow restores an affine-Weyl symmetry and should be responsible for improved properties of the theory under T-duality [38]. Moreover, discrete symmetries analogous to the $Z_{2}$ symmetry $A d S_{5} \leftrightarrow S^{5}$ arise here as well and we have shown that they are symmetries of the quantum theory.

It would be interesting to better understand the similarities of the spectrum with the $A d S_{3}$ case. In this respect, it may be useful to remember that the generators of $S l(2)$ can be constructed in the enveloping algebra of the harmonic oscillator.

We should mention that the vacuum amplitude (this term is more appropriate than partition function since there is no Euclidean continuation) was computed in [19] and found to be equal to that of flat space. There are two potentially non-trivial pieces in this amplitude: the contribution of the zero-level of the current algebra and the existence of null states at $p_{+} \in \mathbb{Z}$. The second is eventually irrelevant since such contributions are of measure zero and $p_{+}$is continuous. In fact this is also true in 
the case of strings in flat Minkowski space-time. At special values of the momenta, there are extra physical states associated to existence of null vectors in the Verma modules. However, they are not visible in the vacuum amplitude.

Summing over the zero-level states (the infinite dimensional reps of $H_{4 L} \times H_{4 R}$ ) gives infinity since they are all degenerate in energy. This is a volume divergence and is analogous to summing over primaries in Minkowski space in a radial coordinate representation of the spectrum. The unambiguous way to sum them is to compute the supergravity finite-time propagator at coincident points (which is also presented in appendix A) and then integrate over the volume.

The exact CFT solution of CW spaces can be written in terms of operators occurring in standard orbifolds of flat space, but for irrational twists. This intriguing fact seems to be related to the observation that such backgrounds are related by formal T-dualities to flat space [17, 18], presumably with identifications. Clarifying this fact should give a better understanding of the free-field resolution of the affine algebra and have further applications. It is conceivable that this remains true also for CW spaces supported by RR backgrounds (relevant for the Penrose limits of near-geometries of D-branes).

One of the main motivations for this study was to try and understand holography in this type of gravitational backgrounds. We have argued that the appropriate patch to carry out this analysis was the analogue of the Poincaré patch of AdS, where the metric is conformal to flat Minkowski space. This patch covers an interval of length $2 \pi$ along the light-cone direction $u$, and the conformal factor depends on $u$ only. The natural holographic direction is then the null light-cone time $x^{+}=-2 \cot (u)$. We have proposed that holography relates S-matrix elements between two holographic screens at $x^{+} \rightarrow \pm \infty$, to off-shell correlation functions in a putative gauge theory dual living on a slice at fixed $x^{+} \rightarrow-\infty$.

The isometries $H_{D L} \times H_{D R}$ of the bulk are realized as a Schrödinger-like representation of the extended Heisenberg algebra on the boundary, whose Ward identities restrict the form of the correlators. We have provided a sample computation of the two- and three-point functions of a massive bulk scalar field. The two-point function seems to vanish while the three-point functions has a non-trivial structure. These quantities should eventually be calculable in string theory. It would be very interesting to have a microscopic definition of the dual theory, to compare our results with. It is unclear at this stage how to construct field theories with the required Heisenberg symmetry.

In contrast to the space-like or time-like holographic set-ups in AdS or dS, the evolution along a null direction is first order in time, which implies that there is a single set of modes at a given mass. These modes are the analogues of the nonnormalizable modes in AdS, corresponding to deformations of the boundary theory. In fact, a careful analysis of the Cauchy problem shows that a complete specification of the field in the bulk requires boundary conditions both at $x^{+}=-\infty$ and $x^{-}=-\infty$. 
Non-trivial boundary values at $x^{-}=-\infty$ give contributions localized at $p_{+}=0$ and are thus associated with the vacuum structure. We have chosen the simplest such boundary conditions (vanishing field at $x^{-}=-\infty$ ) for our computations. Non-trivial boundary conditions here induce divergences at $p_{+}=0$ and it is an open problem to deal with them.

We believe that a better understanding of such backgrounds will shed light on non-space-like versions of holography, and hopefully on holography in flat Minkowski space-time. In addition, it will be interesting to pursue their study from the point of view of time-dependent backgrounds, as they offer interesting solvable exemples of cosmological universes, where the rôle of time is played by a null coordinate.

\section{Acknowledgments}

The authors are grateful to Th. Damour, G. d'Appollonio, J. de Boer, J. Gomis, N. Itzhaki, C. Kounnas, N. Nekrasov, G. Papadopoulos and C. Schweigert for useful discussions. E. K. acknowledges the hospitality of Centre Hospitalier d'Orsay where part of this work was carried out. B. P. thanks the Harvard and U Penn theory groups for hospitality and discussions subsequent to the release of the first draft of this paper. The work of E. K. was partially supported by Marie Curie contract MCFI-2001-0214 and RTN contracts HPRN-CT-2000-00122 and -00131.

Note added. After this article was released, a number of works appeared discussing holography in pp-wave backgrounds from different viewpoints and reaching different conclusions. In [42], by following the AdS/CFT correspondence through the Penrose limit, the holographic direction was argued to be the radial distance in the transverse directions $\mathbb{R}^{p-1}$ originating from the AdS factor in $A d S_{p} \times S^{p}$. In this approach, the holographic degrees of freedom still live in $(p-1)$-dimensional Minkovski space as in the AdS parent, even though the boundary of AdS decouples in the Penrose limit. On the other hand, in [43], the conformal infinity of the pp-wave background is identified as a null trajectory moving along $u$ at infinity in the $\left(v, x_{i}\right)$ space. Based on this, a holographic description as a quantum mechanical model was suggested, in analogy with the M(atrix) model [44]. These different approaches do not necessarily exclude each other, as there may be different holographic descriptions of the same geometry, differing by a possibly complicated change of variables. While all these approaches await a concrete definition of the dual theory, ours makes the symmetries manifest and allows for a direct boundary to bulk correspondence, which we believe is crucial in implementing holography. 


\section{Appendices}

\section{A. Propagators}

In this appendix we will derive and study bulk-to-bulk and bulk-to-boundary propagators for global and conformal coordinates and study their properties. We will

choose boundary conditions such that fields vanish at $v=-\infty$ in global coordinates and at $x^{-}=-\infty$ for conformal coordinates.

\section{A.1 Boundary and bulk to bulk propagators in global coordinates}

The global coordinates were defined in (1.1). For simplicity we will confine ourselves to $H_{4}(\mathrm{D}=4)$. At fixed $p_{+}$, the wave equation in global coordinates is equivalent to the Schrödinger equation for a two-dimensional harmonic oscillator of frequency $\left|p_{+}\right| / 2$. We will use the propagator for a single harmonic oscillator of frequency $\omega$ and mass $m$

$$
\begin{aligned}
G\left(x_{1}, x_{2} ; t\right) & \equiv \sum_{n} \psi_{n}^{*}\left(x_{1}\right) \psi_{n}\left(x_{2}\right) e^{-i E_{n} t} \\
& =\sqrt{\frac{i m \omega}{2 \pi \sin \omega t}} \exp \left[\frac{i m \omega}{\sin \omega t}\left(\frac{1}{2}\left(x_{1}^{2}+x_{2}^{2}\right) \cos \omega t-x_{1} x_{2}\right)\right]
\end{aligned}
$$

normalized so that

$$
\lim _{t \rightarrow 0} G\left(x_{1}, x_{2} ; t\right)=\delta\left(x_{1}-x_{2}\right)
$$

Let $\lambda$ be the eigenvalues of the Laplacian

$$
\Delta F_{\lambda}=\lambda F_{\lambda} \quad, \quad \lambda=-p^{2}-2 p_{+} p_{-}-\left|p_{+}\right|\left(n_{1}+n_{2}+1\right)
$$

with

$$
F_{\lambda}=\exp \left[i\left(p_{+} v+p_{-} u+p \cdot y\right)\right] \psi_{n_{1}}\left(x_{1}\right) \psi_{n_{2}}\left(x_{2}\right)
$$

For a massless mode, the propagator in Schwinger time is therefore

$$
\begin{aligned}
& G\left(u, v, \vec{y}, \vec{x} ; u^{\prime}, v^{\prime}, \vec{y}^{\prime}, \vec{x}^{\prime} ; t\right) \equiv\left\langle u, v, \vec{y}, \vec{x}\left|e^{i t \Delta}\right| u^{\prime}, v^{\prime}, \vec{y}^{\prime}, \vec{x}\right\rangle \\
& =\frac{1}{(2 \pi)^{d+2}} \sum_{n_{1,2}} \int d p_{+} d p_{-} d^{d} p \exp \left[-i p_{+}\left(v-v^{\prime}\right)-i p_{-}\left(u-u^{\prime}\right)-i p \cdot\left(y-y^{\prime}\right)\right] \\
& \times \exp \left[-i t\left(p^{2}+2 p_{+} p_{-}+\left|p_{+}\right|\left(n_{1}+n_{2}+1\right)\right)\right] \psi_{n_{1}, n_{2}}^{*}(x) \psi_{n_{1}, n_{2}}\left(x^{\prime}\right) \\
& =\frac{i}{(2 \pi)^{d+2}} \frac{1}{8 t^{2}}\left(\frac{\pi}{i t}\right)^{d / 2} \frac{u-u^{\prime}}{\sin \left[\left(u-u^{\prime}\right) / 4\right]} \exp \left[\frac{i\left(y-y^{\prime}\right)^{2}}{4 t}\right] \exp \left[\frac{i\left(u-u^{\prime}\right)\left(v-v^{\prime}\right)}{2 t}\right] \times \\
& \times \exp \left[\frac{i\left(u-u^{\prime}\right)}{4 t \sin \left[\left(u-u^{\prime}\right) / 4\right]}\left(\frac{1}{2}\left(x_{1}^{2}+x_{2}^{2}+x_{1}^{\prime 2}+x_{2}^{\prime 2}\right) \cos \left[\left(u-u^{\prime}\right) / 4\right]-x_{1} x_{1}^{\prime}-x_{2} x_{2}^{\prime}\right)\right]
\end{aligned}
$$


where $d$ is the number of transverse flat directions $(d=6$ for the NS5 problem). It satisfies

$$
\lim _{t \rightarrow 0} G\left(u, v, \vec{y}, \vec{x} ; u^{\prime}, v^{\prime}, \vec{y}^{\prime}, \vec{x}^{\prime} ; t\right)=\delta\left(u-u^{\prime}\right) \delta\left(v-v^{\prime}\right) \delta^{(2)}\left(x-x^{\prime}\right) \delta^{(d)}\left(y-y^{\prime}\right)
$$

In the coincidence limit, one obtains

$$
G(u, v, \vec{y}, \vec{x} ; u, v, \vec{y}, \vec{x} ; t)=\frac{1}{2^{d+3} \pi^{d / 2+2} i^{d / 2-1} t^{2+d / 2}}
$$

This value is equal to that of flat $(\mathrm{d}+4)$-dimensional flat space. This was used in [19] in order to compute the vacuum amplitude and show that it is equal to that flat space. The massive bulk propagator is defined as

$$
\left(\Delta-m^{2}\right) H=-\delta\left(u-u^{\prime}\right) \delta\left(v-v^{\prime}\right) \delta^{(2)}\left(x-x^{\prime}\right) \delta^{(d)}\left(y-y^{\prime}\right)
$$

and can be computed from $H\left(z, z^{\prime}\right)=-\sum_{\lambda} F^{*}(z)_{\lambda} F\left(z^{\prime}\right)_{\lambda} /\left(\lambda-m^{2}\right)$. We obtain

$$
\begin{aligned}
H= & \sum_{n_{1}, n_{2}} \int \frac{d p_{+} d p_{-} d^{d} p}{(2 \pi)^{d+2}} \exp \left[-i p_{+}\left(v-v^{\prime}\right)-i p_{-}\left(u-u^{\prime}\right)-i p \cdot\left(y-y^{\prime}\right)\right] \\
& \times \frac{\psi^{*}\left(x_{1}, x_{2}\right)_{n_{1}, n_{2}} \psi\left(x_{1}^{\prime}, x_{2}^{\prime}\right)_{n_{1}, n_{2}}}{p^{2}+2 p_{+} p_{-}+\left|p_{+}\right|\left(n_{1}+n_{2}+1\right)+m^{2}}
\end{aligned}
$$

We perform the contour integral in $p_{-}$and use the oscillator propagator to obtain

$$
\begin{aligned}
& H=\frac{\pi}{4} \int \frac{d p_{+} d^{d} p}{(2 \pi)^{d+2} \sin \left[\left(u-u^{\prime}\right) / 2\right]} \exp \left[-i p_{+}\left(v-v^{\prime}\right)-i p \cdot\left(y-y^{\prime}\right)+i \frac{\left.p^{2}+m^{2}\right)}{2 p_{+}}\left(u-u^{\prime}\right)\right] \\
& \times \exp \left[\frac{i\left|p_{+}\right|}{2 \sin \left[\left(u-u^{\prime}\right) / 2\right]}\left(\frac{1}{2}\left(x_{1}^{2}+x_{2}^{2}+x_{1}^{\prime 2}+x_{2}^{\prime 2}\right) \cos \left[\left(u-u^{\prime}\right) / 2\right]-x_{1} x_{1}^{\prime}-x_{2} x_{2}^{\prime}\right)\right] \\
& =\left(\frac{2 \pi}{-i\left(u-u^{\prime}\right)}\right)^{d / 2} \int \frac{p_{+}^{d / 2} d p_{+}}{8(2 \pi)^{d+1} \sin \left[\left(u-u^{\prime}\right) / 2\right]} \exp \left[-i p_{+}\left(v-v^{\prime}+\frac{1}{2} \frac{\left(y-y^{\prime}\right)^{2}}{u-u^{\prime}}\right)+i \frac{m^{2}\left(u-u^{\prime}\right)}{2 p_{+}}\right] \\
& \times \exp \left[\frac{i\left|p_{+}\right|}{2 \sin \left[\left(u-u^{\prime}\right) / 2\right]}\left(\frac{1}{2}\left(x_{1}^{2}+x_{2}^{2}+x_{1}^{\prime 2}+x_{2}^{\prime 2}\right) \cos \left[\left(u-u^{\prime}\right) / 2\right]-x_{1} x_{1}^{\prime}-x_{2} x_{2}^{\prime}\right)\right]
\end{aligned}
$$

and we finally obtain

$$
H=\frac{1}{4 i^{d-1}(2 \pi)^{(d+2) / 2}} \frac{u-u^{\prime}}{\sin \left[\left(u-u^{\prime}\right) / 2\right]}\left(\frac{m}{r}\right)^{\frac{d+2}{2}} K_{\frac{d+2}{2}}(m r)
$$

where $r$ is the invariant geodesic distance given in (2.9).

\section{A.2 Propagators in conformal coordinates}

Here we work in conformal coordinates

$$
d s^{2}=\frac{1}{f^{2}\left(x^{+}\right)}\left(d x^{+} d x^{-}+\sum d y_{i}^{2}\right), \quad H=\sqrt{f^{\prime \prime} / f^{5}} d x^{+} \wedge \omega
$$


The eigenmodes of the Laplacian

$$
\Delta=f^{2}\left(\Delta_{T}+4 \partial_{+} \partial_{-}\right)-(D-2)\left[f^{2}\right]^{\prime} \partial_{-}
$$

(where $\Delta_{T}=\partial_{i} \partial_{i}$ is the Laplacian on the $(D-2)$ transverse coordinates) are easily computed for arbitrary $f$ profile,

$$
\phi=f^{\frac{D-2}{2}}\left(x^{+}\right) \exp \left(i\left(p_{+} x^{-}+p_{i} y_{i}-\frac{p_{i}^{2}}{4 p_{+}} x^{+}\right)-i \frac{m^{2}}{4 p_{+}} \int_{-\infty}^{x^{+}} \frac{d u}{f^{2}(u)}\right)
$$

We will solve the massive scalar equation $\left(\Delta-m^{2}\right) \phi=0$ and allow for an arbitrary dimension $D$ for the $\mathrm{CW}$ space. Additional flat coordinates can be accommodated by substituting $m^{2} \rightarrow m^{2}+P^{2}$. We first write the solution in terms of the boundary value (at $x^{+}=x^{+}{ }_{0}$ ) in momentum space. We pick also the boundary condition $\phi\left(x^{+},-\infty, y\right)=0$. From now on the integration ranges of $p_{+}$and the $D-2$ transverse momenta $p$ are the whole real line:

$$
\begin{aligned}
\phi_{0}\left(x^{+}, x^{-}, x\right)= & \int d p_{+} d p \chi\left(p_{+}, p\right)\left(\frac{f\left(x^{+}\right)}{f\left(x_{0}^{+}\right)}\right)^{\frac{D-2}{2}} \\
& \times \exp \left[i\left(p_{+} x^{-}+p \cdot x\right)-i \frac{p^{2}}{4 p_{+}}\left(x^{+}-x_{0}^{+}\right)-i \frac{m^{2}}{4 p_{+}} \int_{x_{0}^{+}}^{x^{+}} \frac{d u}{f^{2}(u)}\right]
\end{aligned}
$$

where $\left.\chi) p_{+}, p\right)$ is the Fourier transform of the boundary data at $x^{+}=x_{0}^{+}$,

$$
\lim _{x^{+} \rightarrow x_{0}^{+}} \phi_{0}\left(x^{+}, x^{-}, x\right)=\int d p_{+} d p \chi\left(p_{+}, p\right) \exp \left[i\left(p_{+} x^{-}+p \cdot x\right)\right] \equiv \chi\left(x^{-}, x\right)
$$

From this we can obtain the bulk-to-boundary propagator

$$
\begin{gathered}
K\left(x^{+}, x_{0}^{+} ; x^{-}, x ; x^{-\prime}, x^{\prime}\right)=\int \frac{d p_{+} d p}{(2 \pi)^{D-1}}\left(\frac{f\left(x^{+}\right)}{f\left(x_{0}^{+}\right)}\right)^{\frac{D-2}{2}} \\
\times \exp \left[i\left(p_{+}\left(x^{-}-x^{-\prime}\right)+p \cdot\left(x-x^{\prime}\right)\right)-i \frac{p^{2}}{4 p_{+}}\left(x^{+}-x_{0}^{+}\right)-i \frac{m^{2}}{4 p_{+}} \int_{x_{0}^{+}}^{x^{+}} \frac{d u}{f^{2}(u)}\right]
\end{gathered}
$$

normalized so that

$$
\lim _{x^{+} \rightarrow x_{0}^{+}} K\left(x^{+}, x_{0}^{+} ; x^{-}, x ; x^{-^{\prime}}, x^{\prime}\right)=\delta\left(x^{-}-x^{-^{\prime}}\right) \delta^{(D-2)}\left(x-x^{\prime}\right)
$$

We can perform the momentum integrals in order to obtain the propagator in configuration space,

$$
G\left(x^{+}, x^{-}, y_{i} ; x_{0}^{+}\right)=\theta\left(-r^{2}\right) \frac{\left(x^{+}-x_{0}^{+}\right)^{\frac{(2-D)}{2}}}{(2 \pi)^{D / 2} i^{D-1}}\left(\frac{f\left(x^{+}\right)}{f\left(x_{0}^{+}\right)}\right)^{\frac{D-2}{2}}\left(\frac{\gamma m^{2}}{r^{2}}\right)^{\frac{D}{4}} J_{D / 2}\left(\sqrt{\gamma m^{2} r^{2}}\right)
$$


where $\theta(x)$ is the Heaviside function, $r^{2}$ is the invariant distance from $\left(x^{+}, x^{-}, y_{i}\right)$ to $\left(x_{0}^{+}, 0,0\right)$ in Minkowski space, $r^{2}=\left(x^{+}-x_{0}^{+}\right) x^{-}+y_{1}^{2}+y_{2}^{2}$, and $\gamma$ summarizes the effect of the curved background,

$$
\gamma=\frac{1}{x^{+}-x_{0}^{+}} \int_{x_{0}^{+}}^{x^{+}} \frac{d u}{f^{2}(u)}>0
$$

We would now like to compute the bulk propagator satisfying

$$
\left(\Delta-m^{2}\right) G\left(x^{+}, x^{-}, x ; x^{+^{\prime}}, x^{-^{\prime}}, x^{\prime}\right)=\frac{1}{\sqrt{g}} \delta\left(x^{+}-x^{+^{\prime}}\right) \delta\left(x^{-}-x^{-\prime}\right) \delta^{(D-2)}\left(x-x^{\prime}\right)
$$

Fourier transforming

$$
G\left(x^{+}, x^{-}, x ; x^{+^{\prime}}, x^{-^{\prime}}, x^{\prime}\right)=\int d p_{+} d p e^{i\left(p_{+}\left(x^{-}-x^{-\prime}\right)+p \cdot\left(x-x^{\prime}\right)\right.} G\left(x^{+}, x^{+^{\prime}}, p_{+}, p\right)
$$

we obtain

$$
4 i p_{+} f^{2}\left(x^{+}\right) \partial_{+} G-\left[p^{2} f^{2}\left(x^{+}\right)+m^{2}+i(D-2) p_{+}\left(f^{2}\left(x^{+}\right)\right)^{\prime}\right] G=\frac{f^{D}\left(x^{+}\right)}{(2 \pi)^{D-1}} \delta\left(x^{+}-x^{+^{\prime}}\right)
$$

The solution of the differential equation above can be written as

$G\left(x^{+}, x^{+^{\prime}}, p_{+}, p\right)=\frac{\left[f\left(x^{+}\right) f\left(x^{+^{\prime}}\right)\right]^{\frac{D-2}{2}}}{(2 \pi)^{D-1} 8 i p_{+}} H\left(x^{+}-x^{+^{\prime}}\right) \exp \left[-\frac{i p^{2}}{4 p_{+}}\left(x^{+}-x^{+^{\prime}}\right)-\frac{i m^{2}}{4 p_{+}} \int_{x^{+^{\prime}}}^{x^{+}} \frac{d u}{f^{2}(u)}\right]$

where $H(x)=c \theta(x)+(c-2) \theta(-x) . c=0,2$ corresponds to advanced or retarded propagators. In order to treat symmetrically particles and anti-particles, we choose $c=1$. Performing the momentum integrals we obtain

$$
\begin{aligned}
& G\left(x^{+}, x^{-}, x ; x^{+^{\prime}}, x^{-^{\prime}}, x^{\prime}\right)= \\
& \frac{2^{(D-6) / 2}}{(2 \pi)^{D-1}}\left[f\left(x^{+}\right) f\left(x^{+^{\prime}}\right)\right]^{\frac{D-2}{2}} H\left(x^{+}-x^{+^{\prime}}\right) \times\left(\frac{\pi^{2} m^{2} \gamma}{\left(x^{+}-x_{0}^{+}\right) x^{2}}\right)^{\frac{D-2}{4}} J_{\frac{D-2}{2}}\left(\sqrt{\gamma m^{2} r^{2}}\right)
\end{aligned}
$$

\section{B. The general scalar solution to the wave equation}

In this appendix, we derive the solution $\phi$ of the wave equation $\Delta-m^{2}=0$ in the generalized conformal background (4.3), specified by its values on the wedge $\left(x^{+}=\right.$ $\left.x_{0}^{+}, x^{-}>x_{0}^{-}\right)$and $\left(x^{-}=x_{0}^{-}, x^{+}>x_{0}^{+}\right)$. Defining the normalized field $\phi_{-}=\phi f^{(2-D) / 2}$ and its canonical conjugate $\phi_{+}=\partial_{-} \phi_{-}$, the wave equation can be rewritten in the first order form

$$
\partial_{-} \phi_{-}=\phi_{+}, \quad 4 \partial_{+} \phi_{+}+\Delta_{T} \phi_{-}=\frac{m^{2}}{f^{2}} \phi_{-}
$$


This can be integrated to

$$
\begin{aligned}
& \phi_{-}\left(x^{+}, x^{-}, y\right)=\phi_{-}\left(x_{0}^{-}, x^{+}, y\right)+\int_{x_{0}^{-}}^{x^{-}} d v \phi_{+}\left(x^{+}, v, y\right) \\
& \phi_{+}\left(x^{+}, x^{-}, y\right)=\phi_{+}\left(x_{0}^{+}, x^{-}, y\right)+\frac{1}{4} \int_{x_{0}^{+}}^{x^{+}} d u\left(\frac{m^{2}}{f^{2}(u)}-\Delta_{T}\right) \phi_{-}\left(u, x^{-}, y\right)
\end{aligned}
$$

where $y$ denote collectively the $D-2$ transverse coordinates. Iterating these equations, we get a series expansion for $\phi_{+}$,

$$
\begin{aligned}
\phi_{+}\left(x^{+}, x^{-}, y\right)=\sum_{n=0}^{\infty} \int_{x_{0}^{+}}^{x^{+}} d u_{1} \int_{x_{0}^{+}}^{u_{1}} d u_{2} \ldots \int_{x_{0}^{+}}^{u_{n-1}} d u_{n} \int_{x_{0}^{-}}^{x^{-}} d v_{1} \int_{x_{0}^{-}}^{v_{1}} d v_{2} \ldots \int_{x_{0}^{-}}^{v_{n-1}} d v_{n} \\
\times \prod_{i=1}^{n} \frac{1}{4}\left(\frac{m^{2}}{f^{2}\left(u_{i}\right)}-\Delta_{T}\right)\left[\phi_{+}\left(x_{0}^{+}, v_{n}, y\right)+\frac{1}{4} \int_{x_{0}^{+}}^{u_{n}} d u\left(\frac{m^{2}}{f^{2}(u)}-\Delta_{T}\right) \phi_{-}\left(u, x_{0}^{-}, y\right)\right]
\end{aligned}
$$

We now extend the integration range of $v_{n}$ to $+\infty$ by using the integral representation

$$
\theta\left(v_{n-1}-v_{n}\right)=\int_{-\infty}^{+\infty} \frac{d p_{+}}{2 \pi} \frac{i e^{-i\left(p_{+}+i \epsilon\right)\left(v_{n-1}-v_{n}\right)}}{p_{+}+i \epsilon}
$$

where $\epsilon$ is a positive number unspecified at this stage. Renaming $v=v_{n-1}$ and integrating successively along $v_{n-1}, v_{n-2} \ldots v_{1}$ yields

$$
\begin{aligned}
& \phi_{+}\left(x^{+}, x^{-}, y\right)=\int_{-\infty}^{+\infty} \frac{d p_{+}}{2 \pi} \int_{x_{0}^{-}}^{+\infty} d v e^{i\left(p_{+}+i \epsilon\right)\left(v-x^{-}\right)} \sum_{n=0}^{\infty} \int_{x_{0}^{+}}^{x^{+}} d u_{1} \int_{x_{0}^{+}}^{u_{1}} d u_{2} \ldots \int_{x_{0}^{+}}^{u_{n-1}} d u_{n} \quad \text { (B.6) } \\
&\left(\frac{i}{p_{+}+i \epsilon}\right)^{n} \times \prod_{i=1}^{n} \frac{1}{4}\left(\frac{m^{2}}{f^{2}\left(u_{i}\right)}-\Delta_{T}\right)\left[\phi_{+}\left(x_{0}^{+}, v, y\right)+\frac{1}{4} \int_{x_{0}^{+}}^{u_{n}} d u\left(\frac{m^{2}}{f^{2}(u)}-\Delta_{T}\right) \phi_{-}\left(u, x_{0}^{-}, y\right)\right]
\end{aligned}
$$

Using the identities

$$
\begin{gathered}
\int_{0}^{x^{+}} d u_{1} f\left(u_{1}\right) \int_{0}^{u_{1}} d u_{2} f\left(u_{2}\right) \cdots \int_{0}^{u_{n-1}} d u_{n} f\left(u_{n}\right)=\frac{1}{n !}\left[\int_{0}^{x^{+}} d y f(y)\right]^{n} \\
\int_{0}^{x^{+}} d u_{1} f\left(u_{1}\right) \cdots \int_{0}^{u_{n-1}} d u_{n} f\left(u_{n}\right) \int_{0}^{u_{n}} d u g(u)=\int_{0}^{x^{+}} d u g(u) \frac{1}{n !}\left[\int_{u}^{x^{+}} d y f(y)\right]^{n}
\end{gathered}
$$

one easily obtains the expression (4.19). The conjugate field $\phi_{-}\left(x^{+}, x^{-}, y\right)$ is then obtained by inverting (B.1),

$$
\phi_{-}=\left(4 \partial_{+} \phi_{+}\right) /\left(\frac{m^{2}}{f^{2}}-\Delta_{T}\right)
$$

leading to (4.20) for $\phi_{-}=f^{\frac{(2-D)}{2}} \phi$. Having reached this stage of the computation, it takes little more effort to investigate the issue of particle production in this 
background. The quantization of the scalar field $\phi$ can be carried out by choosing enforcing canonical commutation between the fields $\phi_{+}\left(x_{0}^{+}\right)$and $\phi_{-}\left(x_{0}^{-}\right)$and their canonical conjugate $\partial_{-} \phi_{+}\left(x_{0}^{+}\right)$and $\partial_{+} \phi_{-}\left(x_{0}^{-}\right)$.

$$
\begin{aligned}
& {\left[\phi_{+}\left(x^{+}, x^{-}, x\right), \phi_{+}^{\dagger}\left(x^{+}, x^{-^{\prime}}, x^{\prime}\right)\right]=\delta\left(x^{-}-x^{-^{\prime}}\right) \delta^{(D-2)}\left(x-x^{\prime}\right)} \\
& {\left[\phi_{-}\left(x^{+}, x^{-}, x\right), \phi_{-}^{\dagger}\left(x^{+^{\prime}}, x^{-}, x^{\prime}\right)\right]=\delta\left(x^{+}-x^{+^{\prime}}\right) \delta^{(D-2)}\left(x-x^{\prime}\right)}
\end{aligned}
$$

while $\phi_{+}, \phi_{-}$commute. This follows by imposing the commutation relations on the past wedge and then evolving them to the rest of space-time. Following [31] let us define

$$
\begin{aligned}
& \phi_{+}\left(x^{+}, p_{+}, y\right) \equiv \int_{x_{0}^{-}}^{+\infty} d x^{-} e^{i x^{-} p_{+}} \phi_{+}\left(x^{+}, x^{-}, y\right) \\
& =\int_{x_{0}^{-}}^{+\infty} d v e^{i p_{+} v} \exp \left(\frac{i}{4 p_{+}} \int_{x_{0}^{+}}^{x^{+}}\left(\frac{m^{2}}{f^{2}(u)}-\Delta_{T}\right) d u\right) \phi_{+}\left(x_{0}^{+}, v, y\right) \\
& +\frac{i e^{i p_{+} x_{0}^{-}}}{p_{+}} \times \int_{x_{0}^{+}}^{x^{+}} d u\left[\exp \left(\frac{i}{4 p_{+}} \int_{u}^{x^{+}}\left(\frac{m^{2}}{f^{2}\left(u^{\prime}\right)}-\Delta_{T}\right) d u^{\prime}\right)\right]\left(\frac{m^{2}}{f^{2}(u)}-\Delta_{T}\right) \phi_{-}\left(u, x_{0}^{-}, y\right),
\end{aligned}
$$

where as usual $p_{+} \rightarrow p_{+}+i \epsilon$ is understood, to be the Fourier transform (for $x_{0}^{-} \rightarrow$ $-\infty)$ of the field $\phi_{+}$. We have also used the relation

$$
\int_{x}^{\infty} d z e^{i p z}=\frac{i}{p} e^{i p x}
$$

which follows from $(B .5)$. We can now derive the following relation

$$
\begin{aligned}
-i \partial_{+} \phi_{+}\left(x^{+}, p_{+}, y\right)= & \frac{1}{p_{+}}\left(\frac{m^{2}}{f^{2}(u)}-\Delta_{T}\right) \phi_{+}\left(x^{+}, p_{+}, y\right)+ \\
& +i \frac{e^{i\left(p_{+}+i \epsilon\right) x_{0}^{-}}}{p_{+}+i \epsilon}\left[\frac{m^{2}}{f^{2}\left(x^{+}\right)}-\Delta_{T}\right] \phi_{-}\left(x^{+}, x_{0}^{-}, y\right)
\end{aligned}
$$

We are interested in the limit $\epsilon \rightarrow 0, x_{0}^{-} \rightarrow-\infty$ keeping $\epsilon x_{0}^{-}$constant. Using

$$
\lim _{x_{0}^{-} \rightarrow-\infty, \epsilon \rightarrow 0^{+}} \frac{e^{i\left(p_{+}+i \epsilon\right) x_{0}^{-}}}{p_{+}+i \epsilon}=-2 \pi i \delta\left(p_{+}\right) \quad, \quad \lim _{x_{0}^{-} \rightarrow \infty, \epsilon \rightarrow 0^{+}} \frac{e^{i\left(p_{+}+i \epsilon\right) x_{0}^{-}}}{p_{+}+i \epsilon}=0
$$

we obtain

$$
\begin{aligned}
-i \partial_{+} \phi_{+}\left(x^{+}, p_{+}, y\right)=\frac{1}{p_{+}} & \left(\frac{m^{2}}{f^{2}(u)}-\Delta_{T}\right) \phi_{+}\left(x^{+}, p_{+}, y\right)+ \\
& +2 \pi \delta\left(p_{+}\right)\left[\frac{m^{2}}{f^{2}\left(x^{+}\right)}-\Delta_{T}\right] \phi_{-}\left(x^{+}, x_{0}^{-}, y\right)
\end{aligned}
$$


For $p_{+} \neq 0 \phi_{+}$is an eigenmode of the light-cone Hamiltonian. The sign of its eigenvalue determines whether it is a creation (resp. annihilation) operator. Since $\Delta_{T}<0$, the sign remains that of $p^{+}$irrespective of the $f$-profile. We conclude that there is no particle production of $p_{+} \neq 0$ states. However, this ceases to be true at $p_{+}=0$, due to the second term in $(\mathrm{B} .15)$. At $p_{+}=0$ there is no distinction between particles and anti-particles. Particles can then be produced, as usual in light-cone quantization.

\section{Holography in the Poincaré patch}

In this appendix we provide the calculations for the two- and three-point amplitudes presented in the main body of the paper. We need the solution to massive scalar equation with the appropriate (vanishing) boundary condition at $x^{-}=-\infty$,

$$
\begin{aligned}
\phi_{0}\left(x^{+}, x^{-}, x\right)= & \int d p_{+} d p \chi\left(p_{+}, p\right)\left(\frac{f\left(x^{+}\right)}{f\left(x_{0}^{+}\right)}\right)^{\frac{D-2}{2}} \\
& \times \exp \left[i\left(p_{+} x^{-}+p \cdot x\right)-i \frac{p^{2}}{4 p_{+}}\left(x^{+}-x_{0}^{+}\right)-i \frac{m^{2}}{4 p_{+}} \int_{x_{0}^{+}}^{x^{+}} \frac{d u}{f^{2}(u)}\right]
\end{aligned}
$$

The renormalized source is $\tilde{\chi}=\chi f^{\frac{2-D}{2}}\left(x_{0}^{+}\right)$. The two-point amplitude is given by

$$
\begin{aligned}
F_{2} & =-\frac{1}{2} \int d^{D} x \sqrt{-g}\left(g^{\mu \nu} \partial_{\mu} \phi_{0} \partial_{\nu} \phi_{0}+m^{2} \phi_{0}^{2}\right) \\
& =-\frac{1}{4} \int \frac{d x^{+} d x^{-} d^{D-2} y}{f^{D-2}\left(x^{+}\right)}\left[4 \partial_{+} \phi_{0} \partial_{-} \phi_{0}+\partial_{i} \phi_{0} \partial_{i} \phi_{0}+\frac{m^{2}}{f^{2}} \phi_{0}^{2}\right]=0
\end{aligned}
$$

Here we have substituted the classical solution in the action, and found that, after integrating over $x^{-}, y$, the resulting integrand is zero. The two-point function therefore vanishes.

In order to calculate the three point function, we need the next order solution of the equation of motion.

$$
\left(\Delta-m^{2}\right) \phi_{1}=\phi_{0}^{2}
$$

This can be found from $\phi_{0}$ using the bulk propagator as

$$
\begin{aligned}
\phi_{1}= & \int f^{-D}\left(x^{+^{\prime}}\right) d x^{+^{\prime}} d x^{-^{\prime}} d x^{\prime} G\left(x^{+}, x^{-}, x ; x^{+^{\prime}}, x^{-^{\prime}}, x^{\prime}\right) \phi_{0}^{2}\left(x^{+^{\prime}}, x^{-^{\prime}}, x^{\prime}\right) \\
= & \int d x^{+^{\prime}} \int d p_{+} d q_{+} d p d q \tilde{\chi}\left(p_{+}, p\right) \tilde{\chi}\left(q_{+}, q\right) H\left(x^{+}-x^{+^{\prime}}\right) \frac{1}{8 i\left(p_{+}+q_{+}\right)} \times \\
& \times f\left(x^{+}\right)^{\frac{D-2}{2}} f\left(x^{+^{\prime}}\right)^{\frac{(D-6)}{2}} \exp \left[i\left(p_{+}+q_{+}\right) x^{-}+i(p+q) \cdot x\right] \\
& \times \exp \left[-\frac{i}{4}\left(\frac{p^{2}}{p_{+}}\left(x^{+^{\prime}}-x_{0}^{+}\right)+\frac{q^{2}}{q_{+}}\left(x^{+^{\prime}}-x_{0}^{+}\right)+\frac{(p+q)^{2}}{p_{+}+q_{+}}\left(x^{+}-x^{+^{\prime}}\right)\right)\right] \\
& \times \exp \left[-\frac{i m^{2}}{4}\left(\frac{1}{p_{+}} \int_{x_{0}^{+}}^{x^{+}} \frac{d u}{f^{2}(u)}+\frac{1}{q_{+}} \int_{x_{0}^{+}}^{x^{+}} \frac{d u}{f^{2}(u)}+\frac{1}{p_{+}+q_{+}} \int_{x^{+\prime}}^{x^{+}} \frac{d u}{f^{2}(u)}\right)\right]
\end{aligned}
$$


The three-point amplitude is given by

$$
F_{3}=-\lambda \int d^{D} x \sqrt{-g}\left(g^{\mu \nu} \partial_{\mu} \phi_{0} \partial_{\nu} \phi_{1}+m^{2} \phi_{0} \phi_{1}+\frac{1}{3} \phi_{0}^{3}\right)
$$

We substitute again the solutions into the action do the $x^{+}, x$ integrals and obtain

$$
\begin{aligned}
& \int d^{D} x \sqrt{-g}\left(g^{\mu \nu} \partial_{\mu} \phi_{0} \partial_{\nu} \phi_{1}+m^{2} \phi_{0} \phi_{1}\right)= \\
& =-\frac{1}{4} \int d p_{+} d q_{+} d r_{+} d p d q d r \tilde{\chi}\left(p_{+}, p\right) \tilde{\chi}\left(q_{+}, q\right) \tilde{\chi}\left(r_{+}, r\right) \delta\left(p_{+}+q_{+}+r_{+}\right) \delta(p+q+r) Q_{3}
\end{aligned}
$$

All the terms here cancel point-wise except when a $\partial_{+}$derivative acts on $H\left(x^{+}-x^{+^{\prime}}\right)$ inside $\phi_{1}$. We also obtain

$$
\begin{aligned}
& \frac{1}{3} \int d^{D} x \sqrt{-g} \phi_{0}^{3}= \\
& \quad=\frac{1}{6} \int d p_{+} d q_{+} d r_{+} d p d q d r \tilde{\chi}\left(p_{+}, p\right) \tilde{\chi}\left(q_{+}, q\right) \tilde{\chi}\left(r_{+}, r\right) \delta\left(p_{+}+q_{+}+r_{+}\right) \delta(p+q+r) Q_{3}
\end{aligned}
$$

with

$$
\begin{gathered}
Q_{3}=(2 \pi)^{D-1} \int_{x_{0}^{+}}^{x_{1}^{+}} d u f(u)^{\frac{D-6}{2}} \exp \left[-\frac{i m^{2}}{4}\left(\frac{1}{p_{+}}+\frac{1}{q_{+}}+\frac{1}{r_{+}}\right) \tilde{\gamma}\left(u, x_{0}^{+}\right)\right] \\
\times \exp \left[-\frac{i}{4}\left(\frac{p^{2}}{p_{+}}+\frac{q^{2}}{q_{+}}+\frac{r^{2}}{r_{+}}\right)\left(u-x_{0}^{+}\right)\right]
\end{gathered}
$$

and

$$
\tilde{\gamma}\left(x^{+}, x_{0}^{+}\right) \equiv \int_{x_{0}^{+}}^{x^{+}} \frac{d u}{f^{2}(u)}
$$

Dropping the external sources and the momentum conserving $\delta$-functions we obtain for the three-point function

$$
F_{3}=\frac{\lambda}{12} Q_{3}
$$

\section{Solution of the Ward Identities}

In this section, we study the constraints implied by the algebra of boundary symme- 
tries $H_{D} \times H_{D}$

$$
\begin{aligned}
\tilde{K} & =\partial_{-} \\
\tilde{J}_{L} & =-\frac{y_{1}^{2}+y_{2}^{2}}{2} \partial_{-}+\frac{1}{2}\left(y_{1} \partial_{2}-y_{2} \partial_{1}\right)+\frac{1}{8}\left(4 m^{2}-\Delta\right) \partial_{-}^{-1} \\
\tilde{J}_{R} & =-\frac{y_{1}^{2}+y_{2}^{2}}{2} \partial_{-}-\frac{1}{2}\left(y_{1} \partial_{2}-y_{2} \partial_{1}\right)+\frac{1}{8}\left(4 m^{2}-\Delta\right) \partial_{-}^{-1} \\
\tilde{P}_{i L} & =y_{i} \partial_{-}-\frac{1}{2} \epsilon_{i j} \partial_{j} \\
\tilde{P}_{i R} & =y_{i} \partial_{-}+\frac{1}{2} \epsilon_{i j} \partial_{j}
\end{aligned}
$$

on the correlators of the putative dual gauge theory. The Ward identities for the boundary amplitudes $Q_{n}\left(p_{i}, p_{+}^{i}\right)$ take the form

$$
\sum_{i=1}^{n}\left(\hat{\mathcal{L}}_{a}^{\dagger}\right)_{i} Q_{n}\left(\{p\},\left\{p_{+}\right\}\right)=0
$$

where the operators are the adjoints of those in (D.1). In order for the symmetry to annihilate the boundary "vacuum", its generators should annihilate the part of the classical solution (4.20) that depends on the vacuum data, $\phi\left(x^{+}, x_{0}^{-}, y\right)$. It can be shown that this is true only when $\phi\left(x^{+}, x_{0}^{-}, y\right)=0$ as we assumed.

The constraints on the two-point function have already been discussed in the main text. The $K, P_{L}^{i}-P_{R}^{i}$ identities for the three point function imply momentum conservation. Consequently,

$$
F_{3}\left(p, q, r ; p_{+}, q_{+}, r_{+}\right)=f_{3}\left(p, q, p_{+}, q_{+}\right) \delta\left(p_{+}+q_{+}+r_{+}\right) \delta^{(2)}(p+q+r)
$$

The $P_{L}^{i}+P_{R}^{i}, J_{L}-J_{R}$ identities further imply that

$$
f_{3}\left(p, q, p_{+}, q_{+}\right)=g\left(p_{+}, q_{+}, z\right)
$$

where

$$
z=\left(\frac{p}{p_{+}}-\frac{q}{q_{+}}\right)^{2}=\left(\frac{1}{p_{+}}+\frac{1}{q_{+}}\right)\left(\frac{p^{2}}{p_{+}}+\frac{q^{2}}{q_{+}}+\frac{r^{2}}{r_{+}}\right)
$$

Finally, the $J_{L}+J_{R}$ identity leads to the differential equation $g$

$$
\left[\partial_{z}^{2}+\frac{1}{z} \partial_{z}-\frac{B}{4 A z}-\frac{1}{16 A^{2}}\right] g=0
$$

where

$$
A=\frac{1}{p_{+}}+\frac{1}{q_{+}} \quad, \quad B=\frac{m_{1}^{2}}{p_{+}}+\frac{m_{2}^{2}}{q_{+}}+\frac{m_{3}^{2}}{r_{+}}
$$

This equation is related to the confluent hypergeometric equation. The two linearly independent solutions are

$$
g_{+}=e^{-\frac{z}{4 A}} F\left(\frac{(1+B}{2}, 1, \frac{z}{2 A}\right)
$$




$$
g_{-}=e^{-\frac{z}{4 A}} U\left(\frac{(1+B}{2}, 1, \frac{z}{2 A}\right)
$$

where $F(a, b, x)$ is Kummer's confluent hypergeometric function

$$
F(a, b, x) \equiv 1+\frac{a}{b} \frac{x}{1 !}+\frac{a(a+1)}{b(b+1)} \frac{x^{2}}{2 !}+\cdots
$$

and $U(a, b, x)$ is the linearly independent conjugate Kummer's function. In our case it is logarithmic

$$
U(a, 1, x)=-\frac{1}{\Gamma(a)}\left[\frac{\partial}{\partial a} F(a, 1, x)+\left(2 \gamma_{E}+\psi(a)+\log x\right) F(a, 1, x)\right]
$$

We also have the integral representations

$$
\begin{gathered}
F(a, b, x)=\frac{\Gamma(b)}{\Gamma(a) \Gamma(b-a)} \int_{0}^{1} d t e^{z t} t^{a-1}(1-t)^{b-a-1} \\
U(a, b, x)=\frac{1}{\Gamma(a)} \int_{0}^{\infty} d t e^{-z t} t^{a-1}(1+t)^{b-a-1}
\end{gathered}
$$

Using the asymptotic limits

$$
\begin{gathered}
F(a, b, x)=\frac{\Gamma(b)}{\Gamma(a)} x^{a-b} e^{x}\left[1+\mathcal{O}\left(\frac{1}{x}\right)\right] \\
U(a, b, x)=x^{-a}\left[1+\mathcal{O}\left(\frac{1}{x}\right)\right]
\end{gathered}
$$

we obtain

$$
g_{ \pm} \sim z^{\frac{-1 \pm B}{2}} e^{ \pm \frac{z}{4 A}} \quad, \quad z \rightarrow \infty
$$

Thus, the general solution to the Ward identities for the 3-point amplitude is given by

$$
\begin{aligned}
f_{3}\left(p, q ; p_{+}, q_{+}\right)= & \exp \left[-\frac{z}{4 A}\right]\left[C_{+}(A, B) F\left(\frac{(1+B}{2}, 1, \frac{z}{2 A}\right)+\right. \\
& \left.+C_{-}(A, B) U\left(\frac{(1+B)}{2}, 1, \frac{z}{2 A}\right)\right]
\end{aligned}
$$

It is easy to see that our three-point amplitude for the scalar theory satisfies the appropriate second order equation and is thus of this form. For the $N$-point function, the solution to all but the $J_{L}+J_{R}$ Ward identity can be written as

$$
F_{n}\left(p_{I}, p_{+}^{I}\right)=f_{n}\left(z^{i}, \hat{z}^{\alpha}, p_{+}^{K}\right) \delta\left(\sum_{I=1}^{n} p_{+}^{I}\right) \delta^{(2)}\left(\sum_{I=1}^{n} p_{I}\right)
$$

where

$$
\begin{aligned}
z^{i} & =\frac{p_{1}^{2}}{p_{+}^{1}}+\frac{p_{2}^{2}}{p_{+}^{2}}+\frac{p_{i}^{2}}{p_{+}^{i}} \quad, \quad i=3,4, \cdots, n \\
\hat{z}^{\alpha} & =\frac{p_{1}^{2}}{p_{+}^{1}}+\frac{p_{3}^{2}}{p_{+}^{3}}+\frac{p_{\alpha}^{2}}{p_{+}^{\alpha}} \quad, \quad \alpha=4,5, \cdots, n
\end{aligned}
$$

Finally the $J_{L}+J_{R}$ Ward identity implies a second order partial differential equation on the variables $z^{i}, \hat{z}^{\alpha}$. 


\section{E. Holography in the harmonic oscillator basis}

In this appendix we translate our holographic derivation of boundary correlators to the (global) harmonic oscillator basis. At fixed $p_{+}$the transverse coordinates are effectively compact. We can therefore reduce the wave equation à la Kaluza-Klein. The analogue of the spherical harmonics are the modes

$$
D_{p_{+}, n_{1}, n_{2}}\left(v, x^{i}\right)=\frac{1}{\sqrt{2 \pi}} e^{i p_{+} v} \psi_{n_{1}}\left(x^{1}\right) \psi_{n_{2}}\left(x^{2}\right)
$$

where $\psi_{n}$ are the normalized harmonic oscillator wave-functions with frequency $\omega=$ $\left|p_{+}\right| / 2$,

$$
\int d v d^{2} x D_{p_{+}, n_{1}, n_{2}}^{*}\left(v, x^{i}\right) D_{p_{+}^{\prime}, n_{1}^{\prime}, n_{2}^{\prime}}^{*}\left(v, x^{i}\right)=\delta\left(p_{+}-p_{+}^{\prime}\right) \delta_{n_{1}, n_{1}^{\prime}} \delta_{n_{2}, n_{2}^{\prime}}
$$

We make the decomposition

$$
\phi\left(u, v, x^{i}\right)=\sum_{n_{1,2}=0}^{\infty} \int_{-\infty}^{\infty} d p_{+} \xi_{p_{+}, n_{1}, n_{2}}(u) D_{p_{+}, n_{1}, n_{2}}\left(v, x^{i}\right)
$$

The Laplacian equation for a complex massive scalar

$$
\left(2 \partial_{u} \partial_{v}+\frac{|x|^{2}}{4} \partial_{v}^{2}+\partial_{1}^{2}+\partial_{2}^{2}-m^{2}\right) \phi=0
$$

becomes

$$
\partial_{u} \xi_{p_{+}, n_{1}, n_{2}}=-\frac{i}{2 p_{+}}\left(m^{2}+\left|p_{+}\right|\left(n_{1}+n_{2}+1\right)\right) \xi_{p_{+}, n_{1}, n_{2}}
$$

Consider a complex scalar scalar field with free action

$$
S=\frac{1}{2} \int d u d v d^{2} x\left[\partial_{u} \phi \partial_{v} \phi^{*}+\partial_{u} \phi^{*} \partial_{v} \phi+\frac{|x|^{2}}{4}\left|\partial_{v} \phi\right|^{2}+\left|\partial_{i} \phi\right|^{2}+m^{2}|\phi|^{2}\right]
$$

We "compactify" using the decomposition (E.3). For this we need the matrix elements $\left\langle m\left|x^{2}\right| n\right\rangle$ and $\left\langle m\left|\partial_{x}^{2}\right| n\right\rangle$ for a single harmonic oscillator

$$
\begin{aligned}
\left\langle m\left|x^{2}\right| n\right\rangle & =\frac{1}{2 \omega} X_{m, n} \equiv \frac{1}{2 \omega}\left[\sqrt{n(n-1)} \delta_{m, n-2}+(2 n+1) \delta_{m, n}+\sqrt{(n+1)(n+2)} \delta_{m, n+2}\right] \\
\left\langle m\left|\partial_{x}^{2}\right| n\right\rangle & =\frac{\omega}{2} P_{m, n} \equiv \frac{\omega}{2}\left[\sqrt{n(n-1)} \delta_{m, n-2}-(2 n+1) \delta_{m, n}+\sqrt{(n+1)(n+2)} \delta_{m, n+2}\right]
\end{aligned}
$$

The action of the symmetry in this basis can be inferred from the following: For a two-dimensional symmetric harmonic oscillator we can write the angular momentum as

$$
L=\frac{i}{2}\left(x^{1} \partial_{2}-x^{2} \partial_{1}\right)=\frac{i}{2}\left(a_{2} a_{1}^{\dagger}-a_{1} a_{2}^{\dagger}\right)
$$


The spectrum of $L$ for states $|n, N-n\rangle$ takes the values $-\frac{N}{2} \leq L \leq \frac{N}{2}$ and is integerspaced. Thus, states can be classified by their energy $\omega(N+1)$ and the angular momentum $L$. Since

$$
\left[L, a_{1} \pm i a_{2}\right]=\mp \frac{1}{2}\left(a_{1} \pm a_{2}\right) \quad, \quad\left[L, a_{1}^{\dagger} \pm i a_{2}^{\dagger}\right]=\mp \frac{1}{2}\left(a_{1}^{\dagger} \pm a_{2}^{\dagger}\right)
$$

we obtain

$$
\begin{aligned}
& \left(a_{1} \pm i a_{2}\right)|N, L\rangle=\sqrt{N \pm 2 L}\left|N-1, L \mp \frac{1}{2}\right\rangle \\
& \left(a_{1}^{\dagger} \pm i a_{2}^{\dagger}\right)|N, L\rangle=\sqrt{N+2 \mp 2 L}\left|N+1, L \mp \frac{1}{2}\right\rangle
\end{aligned}
$$

where $\left\langle N, L \mid N^{\prime}, L^{\prime}\right\rangle=\delta_{N, N^{\prime}} \delta_{L+L^{\prime}}$. The action can be further evaluated using

$$
\begin{gathered}
\int d v d^{2} x D_{p_{+}, n_{1}, n_{2}}^{*} \partial_{v} D_{p_{+}^{\prime}, n_{1}^{\prime}, n_{2}^{\prime}}=i p_{+} \delta\left(p_{+}-p_{+}^{\prime}\right) \delta_{n_{1}, n_{1}^{\prime}} \delta_{n_{2}, n_{2}^{\prime}} \\
\int d v d^{2} x \frac{|x|^{2}}{4} \partial_{v} D_{p_{+}, n_{1}, n_{2}}^{*} \partial_{v} D_{p_{+}^{\prime}, n_{1}^{\prime}, n_{2}^{\prime}}=\frac{\left|p_{+}\right|}{4} \delta\left(p_{+}-p_{+}^{\prime}\right)\left[\delta_{n_{1}, n_{1}^{\prime}} X_{n_{2}, n_{2}^{\prime}}+\delta_{n_{2}, n_{2}^{\prime}} X_{n_{1}, n_{1}^{\prime}}\right] \\
\int d v d^{2} x\left[\partial_{x^{1}} D_{p_{+}, n_{1}, n_{2}}^{*} \partial_{x^{1}} D_{p_{+}^{\prime}, n_{1}^{\prime}, n_{2}^{\prime}}+\partial_{x^{2}} D_{p_{+}, n_{1}, n_{2}}^{*} \partial_{x^{2}} D_{p_{+}^{\prime}, n_{1}^{\prime}, n_{2}^{\prime}}\right] \\
=-\frac{\left|p_{+}\right|}{4} \delta\left(p_{+}-p_{+}^{\prime}\right)\left[\delta_{n_{1}, n_{1}^{\prime}} P_{n_{2}, n_{2}^{\prime}}+\delta_{n_{2}, n_{2}^{\prime}} P_{n_{1}, n_{1}^{\prime}}\right]
\end{gathered}
$$

Using the above the action becomes

$$
\begin{aligned}
S= & \frac{1}{2} \sum_{n_{1,2}=0}^{\infty} \int_{-\infty}^{\infty} d p_{+} \int d u\left[2 p_{+} \operatorname{Im}\left(\partial_{u} \xi_{p_{+}, n_{1}, n_{2}} \xi_{p_{+}, n_{1}, n_{2}}^{*}\right)+m^{2}\left|\xi_{p_{+}, n_{1}, n_{2}}\right|^{2}\right]+(\mathrm{E} .13) \\
+ & \frac{1}{8} \sum_{n_{1,2}, m_{1,2}=0}^{\infty} \int_{-\infty}^{\infty} d p_{+} \int d u\left|p_{+}\right| \xi_{p_{+}, n_{1}, n_{2}}^{*} \xi_{p_{+}, m_{1}, m_{2}}\left[\delta_{n_{1}, m_{1}}(X-P)_{n_{2}, m_{2}}+\delta_{n_{2}, m_{2}}(X-P)_{n_{1}, m_{1}}\right] \\
= & \frac{1}{2} \sum_{n_{1,2}=0}^{\infty} \int_{-\infty}^{\infty} d p_{+} \int d u\left[2 p_{+} \operatorname{Im}\left(\partial_{u} \xi_{p_{+}, n_{1}, n_{2}} \xi_{p_{+}, n_{1}, n_{2}}^{*}\right)+\left(m^{2}+\left|p_{+}\right|\left(n_{1}+n_{2}+1\right)\right)\left|\xi_{p_{+}, n_{1}, n_{2}}\right|^{2}\right]
\end{aligned}
$$

Evaluating the action on the classical solution

$$
\xi_{p_{+}, n_{1}, n_{2}}(u)=\zeta_{p_{+}, n_{1}, n_{2}} \exp \left[-\frac{i}{2 p_{+}}\left(m^{2}+\left|p_{+}\right|\left(n_{1}+n_{2}+1\right)\right) u\right]
$$

we obtain

$$
S=\frac{V_{u}}{2} \sum_{n_{1,2}=0}^{\infty} \int_{-\infty}^{\infty} d p_{+}\left[\left(2 p_{+} p_{-}+m^{2}+2\left|p_{+}\right|\left(n_{1}+n_{2}+1\right)\right)\left|\zeta_{p_{+}, n_{1}, n_{2}}\right|^{2}\right]=0
$$

where $V_{u}$ is the (infinite) volume of the $u$-line and

$$
p_{-}=-\frac{1}{2 p_{+}}\left(m^{2}+\left|p_{+}\right|\left(n_{1}+n_{2}+1\right)\right)
$$


Thus, as in Appendix C, two-point interactions of the boundary sources vanish.

We will now consider the cubic interaction of the form

$$
S_{\text {int }}=g \int d u d v d^{2} x \phi_{1} \phi_{2} \phi_{3}
$$

where $\phi_{i}$ are real scalars with masses $m_{i}$. Define the overlap integral of harmonic oscillator wave-functions

$$
\int_{-\infty}^{\infty} d x \psi_{n_{1}}^{\omega_{1}}(x) \psi_{n_{2}}^{\omega_{2}}(x) \psi_{n_{3}}^{\omega_{3}}(x)=C_{n_{1}, n_{2}, n_{3}}\left(\omega_{1}, \omega_{2}, \omega_{3}\right)
$$

It can be evaluated from the generating function

$$
\begin{aligned}
& C\left(t_{1}, t_{2}, t_{3} ; \omega_{1}, \omega_{2}, \omega_{3}\right) \equiv \sum_{n_{1}, n_{2}, n_{3}=0}^{\infty} \frac{\left(\sqrt{2} t_{1}\right)^{n_{1}}\left(\sqrt{2} t_{2}\right)^{n_{2}}\left(\sqrt{2} t_{3}\right)^{n_{3}}}{\sqrt{n_{1} ! n_{2} ! n_{3} !}} C_{n_{1}, n_{2}, n_{3}}\left(\omega_{1}, \omega_{2}, \omega_{3}\right) \\
= & \left(\frac{4 \omega_{1} \omega_{2} \omega_{3}}{\pi\left(\omega_{1}+\omega_{2}+\omega_{3}\right)^{2}}\right)^{\frac{1}{4}} \exp \left[2 \frac{\left(\sqrt{\omega_{1}} t_{1}+\sqrt{\omega_{2}} t_{2}+\sqrt{\omega_{3}} t_{3}\right)^{2}}{\omega_{1}+\omega_{2}+\omega_{3}}-\frac{1}{2}\left(t_{1}^{2}+t_{2}^{2}+t_{3}^{2}\right)\right]
\end{aligned}
$$

We obtain for the three-point amplitude

$$
\begin{aligned}
S_{i n t} & =g \sqrt{2 \pi} \sum_{n_{1,2}^{i}=0}^{\infty} \int\left[\prod_{i=1}^{3} d p_{+}^{i} \zeta_{p_{+}^{i}, n_{1}^{i}, n_{2}^{i}}\right] \delta\left(p_{-}^{1}+p_{-}^{2}+p_{-}^{3}\right) \delta\left(p_{+}^{1}+p_{+}^{2}+p_{+}^{3}\right) \times \\
& \times C_{n_{1}^{1}, n_{1}^{2}, n_{1}^{3}}\left(\left|p_{+}^{1}\right|,\left|p_{+}^{2}\right|,\left|p_{+}^{3}\right|\right) C_{n_{2}^{1}, n_{2}^{2}, n_{2}^{3}}\left(\left|p_{+}^{1}\right|,\left|p_{+}^{2}\right|,\left|p_{+}^{3}\right|\right)
\end{aligned}
$$

Higher point amplitudes can be computed along similar lines.

\section{References}

[1] O. Aharony, S. S. Gubser, J. Maldacena, H. Ooguri and Y. Oz, "Large N field theories, string theory and gravity," Phys. Rept. 323, 183 (2000) [arXiv:hep-th/9905111]; R. Bousso, "The holographic principle," arXiv:hep-th/0203101.

[2] R. Penrose, "Any space-time has a plane wave as a limit", in Differential geometry and relativity, Riedel, Dordrecht, 1976.

[3] M. Blau, J. Figueroa-O'Farrill, C. Hull and G. Papadopoulos, "Penrose limits and maximal supersymmetry," arXiv:hep-th/0201081;

[4] M. Blau, J. Figueroa-O'Farrill, C. Hull and G. Papadopoulos, "A new maximally supersymmetric background of IIB superstring theory," JHEP 0201, 047 (2002) [arXiv:hep-th/0110242].

[5] R. R. Metsaev, "Type IIB Green-Schwarz superstring in plane wave Ramond-Ramond background," Nucl. Phys. B 625, 70 (2002) [arXiv:hep-th/0112044]; R. R. Metsaev and A. A. Tseytlin, "Exactly solvable model of superstring in plane wave RamondRamond background," arXiv:hep-th/0202109. 
[6] D. Berenstein, J. Maldacena and H. Nastase, "Strings in flat space and pp waves from N = 4 super Yang Mills," arXiv:hep-th/0202021.

[7] M. Hatsuda, K. Kamimura and M. Sakaguchi, "From super-AdS(5) x S**5 algebra to super-pp-wave algebra," arXiv:hep-th/0202190.

[8] R. Gopakumar, J. Maldacena, S. Minwalla and A. Strominger, "S-duality and noncommutative gauge theory," JHEP 0006, 036 (2000) [arXiv:hep-th/0005048].

[9] J. Kowalski-Glikman, "Vacuum States In Supersymmetric Kaluza-Klein Theory," Phys. Lett. B 134, 194 (1984).

[10] C. Bachas, J. Hoppe and B. Pioline, "Nahm equations, $N=1^{*}$ domain walls, and D-strings in AdS(5) x S(5)," JHEP 0107, 041 (2001) [arXiv:hep-th/0007067].

[11] N. Itzhaki, I. R. Klebanov and S. Mukhi, "PP wave limit and enhanced supersymmetry in gauge theories," arXiv:hep-th/0202153; L. A. Zayas and J. Sonnenschein, "On Penrose limits and gauge theories," arXiv:hep-th/0202186.

[12] J. Gomis and H. Ooguri, "Penrose limit of $N=1$ gauge theories," arXiv:hepth/0202157.

[13] M. Blau, J. Figueroa-O'Farrill and G. Papadopoulos, "Penrose limits, supergravity and brane dynamics," arXiv:hep-th/0202111; M. Alishahiha and M. M. Sheikh-Jabbari, "The PP-wave limits of orbifolded AdS(5) x S**5," arXiv:hep-th/0203018; N. w. Kim, A. Pankiewicz, S. J. Rey and S. Theisen, "Superstring on pp-wave orbifold from large-N quiver gauge theory," arXiv:hep-th/0203080; M. Cvetic, H. Lu and C. N. Pope, "Penrose limits, pp-waves and deformed M2-branes," arXiv:hep-th/0203082; T. Takayanagi and S. Terashima, "Strings on orbifolded pp-waves," arXiv:hep-th/0203093; U. Gursoy, C. Nunez and M. Schvellinger, "RG flows from Spin(7), CY 4-fold and HK manifolds to AdS, Penrose limits and pp waves," arXiv:hep-th/0203124; E. Floratos and A. Kehagias, "Penrose limits of orbifolds and orientifolds," arXiv:hep-th/0203134; M. Cvetic, H. Lu and C. N. Pope, "M-theory pp-waves, Penrose limits and supernumerary supersymmetries," arXiv:hep-th/0203229. J. P. Gauntlett and C. M. Hull, "pp-waves in 11-dimensions with extra supersymmetry," arXiv:hep-th/0203255.

[14] C. Kounnas, M. Porrati and B. Rostand, "On N=4 Extended Superliouville Theory," Phys. Lett. B 258, 61 (1991); C. G. Callan, J. A. Harvey and A. Strominger, "Worldbrane actions for string solitons," Nucl. Phys. B 367, 60 (1991).

[15] C. R. Nappi and E. Witten, "A WZW model based on a nonsemisimple group," Phys. Rev. Lett. 71, 3751 (1993) [arXiv:hep-th/9310112].

[16] "Gauged WZW models and nonAbelian duality," Phys. Rev. D 50 (1994) 2784 [arXiv:hep-th/9402031]; A. A. Kehagias and P. A. Meessen, "Exact string background from a WZW model based on the Heisenberg group," Phys. Lett. B 331, 77 (1994) [arXiv:hep-th/9403041]. 
[17] E. Kiritsis and C. Kounnas, "String Propagation In Gravitational Wave Backgrounds," Phys. Lett. B 320, 264 (1994) [Addendum-ibid. B 325, 536 (1994)] [arXiv:hepth/9310202].

[18] C. Klimcik and A. A. Tseytlin, "Duality invariant class of exact string backgrounds," Phys. Lett. B 323 (1994) 305 [arXiv:hep-th/9311012];

[19] E. Kiritsis, C. Kounnas and D. Lust, "Superstring gravitational wave backgrounds with space-time supersymmetry," Phys. Lett. B 331, 321 (1994) [arXiv:hepth/9404114].

[20] R. Güven, "Plane Waves In Effective Field Theories Of Superstrings," Phys. Lett. B 191 (1987) 275; D. Amati and C. Klimcik, "Strings In A Shock Wave Background And Generation Of Curved Geometry From Flat Space String Theory," Phys. Lett. B 210 (1988) 92; "Nonperturbative Computation Of The Weyl Anomaly For A Class Of Nontrivial Backgrounds," Phys. Lett. B 219 (1989) 443; H. J. de Vega and N. Sanchez, "Particle Scattering At The Planck Scale And The Aichelburg-Sexl Geometry," Nucl. Phys. B 317 (1989) 731; G. T. Horowitz and A. R. Steif, "Space-Time Singularities In String Theory," Phys. Rev. Lett. 64 (1990) 260; "Strings In Strong Gravitational Fields," Phys. Rev. D 42 (1990) 1950; A. R. Steif, "Nonperturbative Time Dependent Classical String Solutions For The Closed Bosonic String," Phys. Rev. D 42 (1990) 2150; A. A. Tseytlin, "A Class of finite two-dimensional sigma models and string vacua," Phys. Lett. B 288 (1992) 279 [arXiv:hep-th/9205058]; "String vacuum backgrounds with covariantly constant null Killing vector and 2-d quantum gravity," Nucl. Phys. B 390 (1993) 153 [arXiv:hep-th/9209023]; "Finite sigma models and exact string solutions with Minkowski signature metric," Phys. Rev. D 47 (1993) 3421 [arXiv:hep-th/9211061] C. Klimcik and A. A. Tseytlin, "Duality invariant class of exact string backgrounds," Phys. Lett. B 323 (1994) 305 [arXiv:hep-th/9311012]; "Exact four-dimensional string solutions and Toda like sigma models from 'null gauged' WZNW theories," , Nucl. Phys. B 424 (1994) 71 [arXiv:hep-th/9402120]; K. Sfetsos, "Gauging a nonsemisimple WZW model," Phys. Lett. B 324 (1994) 335 [arXiv:hepth/9311010]; "Exact string backgrounds from WZW models based on nonsemisimple groups," Int. J. Mod. Phys. A 9 (1994) 4759 [arXiv:hep-th/9311093]; N. Mohammedi, "On bosonic and supersymmetric current algebras for nonsemisimple groups," Phys. Lett. B 325 (1994) 371 [arXiv:hep-th/9312182]; A. Kumar and S. Mahapatra, "Exact duality and nilpotent gauging," Mod. Phys. Lett. A 9 (1994) 925 [arXiv:hepth/9401098]; J. M. Figueroa-O'Farrill and S. Stanciu, "Nonsemisimple Sugawara constructions," Phys. Lett. B 327 (1994) 40 [arXiv:hep-th/9402035]; I. Antoniadis and N. A. Obers, "Plane gravitational waves in string theory," Nucl. Phys. B 423 (1994) 639 [arXiv:hep-th/9403191]; K. Sfetsos and A. A. Tseytlin, "Four-dimensional plane wave string solutions with coset CFT description," Nucl. Phys. B 427 (1994) 245 [arXiv:hep-th/9404063].

[21] O. Jofre and C. Nunez, "Strings In Plane Wave Backgrounds Revisited," Phys. Rev. D 50 (1994) 5232 [arXiv:hep-th/9311187]; 
[22] J. G. Russo and A. A. Tseytlin, "On solvable models of type IIB superstring in NS-NS and R-R plane wave backgrounds," arXiv:hep-th/0202179.

[23] J. G. Russo and A. A. Tseytlin, "Green-Schwarz superstring action in a curved magnetic Ramond-Ramond background," JHEP 9804, 014 (1998) [arXiv:hep-th/9804076].

[24] J. G. Russo and A. A. Tseytlin, "Constant magnetic field in closed string theory: An Exactly solvable model," Nucl. Phys. B 448, 293 (1995) [arXiv:hep-th/9411099].

[25] E. Witten, "Quantum gravity in de Sitter space," arXiv:hep-th/0106109.

[26] A. Strominger, "The ds/CFT correspondence," JHEP 0110, 034 (2001) [arXiv:hepth/0106113].

[27] E. Witten, talk at Strings 1998, ITP, available at http://online.itp.ucsb.edu/online/strings98/witten/

[28] R. F. Streater, "The representations of the Oscillator group", Comm. Math. Phys. 4 (1967) 217.

[29] M. B. Halpern and E. Kiritsis, "General Virasoro Construction On Affine G," Mod. Phys. Lett. A 4 (1989) 1373.

[30] J. G. Russo, "Thermal ensemble of string gas in a magnetic field," Phys. Lett. B 335, 168 (1994) [arXiv:hep-th/9405118].

[31] T. N. Tomaras, N. C. Tsamis and R. P. Woodard, "Pair creation and axial anomaly in light-cone QED(2)," JHEP 0111, 008 (2001) [arXiv:hep-th/0108090]; T. N. Tomaras, N. C. Tsamis and R. P. Woodard, "Back-reaction in lightcone QED," Phys. Rev. D 62, 125005 (2000) [arXiv:hep-ph/0007166].

[32] P. Forgacs, P. A. Horvathy, Z. Horvath and L. Palla, "The Nappi-Witten string in the light-cone gauge," Heavy Ion Phys. 1, 65 (1995) [arXiv:hep-th/9503222].

[33] D. I. Olive, E. Rabinovici and A. Schwimmer, "A Class of string backgrounds as a semiclassical limit of WZW models," Phys. Lett. B 321, 361 (1994) [arXiv:hepth/9311081].

[34] S. Stanciu and A. A. Tseytlin, "D-branes in curved spacetime: Nappi-Witten background," JHEP 9806, 010 (1998) [arXiv:hep-th/9805006].

[35] J. M. Figueroa-O'Farrill and S. Stanciu, "More D-branes in the Nappi-Witten background," JHEP 0001, 024 (2000) [arXiv:hep-th/9909164].

[36] S. R. Das, C. Gomez and S. J. Rey, "Penrose limit, spontaneous symmetry breaking and holography in pp-wave background," arXiv:hep-th/0203164.

[37] J. Maldacena and H. Ooguri, "Strings in $\operatorname{AdS}(3)$ and SL(2,R) WZW model. I," J. Math. Phys. 42 (2001) 2929 [arXiv:hep-th/0001053];

J. Maldacena, H. Ooguri and J. Son, "Strings in AdS(3) and the SL(2,R) WZW model. II: Euclidean black hole," J. Math. Phys. 42 (2001) 2961 [arXiv:hep-th/0005183]. 
[38] E. B. Kiritsis, "Duality in gauged WZW models," Mod. Phys. Lett. A 6 (1991) 2871; "Exact duality symmetries in CFT and string theory," Nucl. Phys. B 405 (1993) 109 [arXiv:hep-th/9302033].

[39] S. N. Solodukhin, "Conformal description of horizon's states," Phys. Lett. B 454, 213 (1999) [arXiv:hep-th/9812056]; I. Sachs and S. N. Solodukhin, "Horizon holography," Phys. Rev. D 64, 124023 (2001) [arXiv:hep-th/0107173].

[40] V. Balasubramanian, P. Kraus and A. E. Lawrence, "Bulk vs. boundary dynamics in anti-de Sitter spacetime," Phys. Rev. D 59, 046003 (1999) [arXiv:hep-th/9805171]; S. de Haro, S. N. Solodukhin and K. Skenderis, "Holographic reconstruction of spacetime and renormalization in the AdS/CFT correspondence," Commun. Math. Phys. 217 (2001) 595 [arXiv:hep-th/0002230].

[41] S. S. Gubser, I. R. Klebanov and A. M. Polyakov, "Gauge theory correlators from non-critical string theory," Phys. Lett. B 428, 105 (1998) [arXiv:hep-th/9802109]. E. Witten, "Anti-de Sitter space and holography," Adv. Theor. Math. Phys. 2, 253 (1998) [arXiv:hep-th/9802150];

[42] R. G. Leigh, K. Okuyama and M. Rozali, "PP-waves and holography," arXiv:hepth/0204026.

[43] D. Berenstein and H. Nastase, "On lightcone string field theory from super Yang-Mills and holography," arXiv:hep-th/0205048.

[44] T. Banks, W. Fischler, S. H. Shenker and L. Susskind, "M theory as a matrix model: A conjecture," Phys. Rev. D 55, 5112 (1997) [arXiv:hep-th/9610043]. 